\title{
Three stellar associations and their field east of LMC 4 in the Large Magellanic Cloud
}

\author{
D. Gouliermis ${ }^{1}$, S. C. Keller ${ }^{2}$, K. S. de Boer ${ }^{1}$, M. Kontizas ${ }^{3}$, and E. Kontizas ${ }^{4}$ \\ 1 Sternwarte der Universität Bonn, Auf dem Hügel 71, 53121 Bonn, Germany \\ 2 Institute of Geophysics and Planetary Physics, Lawerence Livermore National Laboratory, 413, PO Box 808, \\ Livermore, CA 94550, USA \\ 3 Department of Astrophysics Astronomy \& Mechanics, Faculty of Physics, University of Athens, \\ 15783 Athens, Greece \\ 4 Institute for Astronomy and Astrophysics, National Observatory of Athens, PO Box 20048, \\ 11810 Athens, Greece
}

Received 20 December 2000 / Accepted 18 October 2001

\begin{abstract}
We present BVR photometry in an area of $20.5 \times 20^{\prime} .5$ centered on LH 95 situated to the north-east of the super-bubble LMC 4. We investigate the stellar content of three stellar associations (LH 91, LH 91-I \& LH 95) and their surrounding fields. Our observations use the $R-\mathrm{H} \alpha$ colour index to identify the Be star population of the region. We find that Be stars exist in all three of the investigated associations. Within LH 95 we find a central cluster of four Be stars which strongly determine the HII emissivity in this area. We estimated the reddening and the age of the systems based on isochrone fitting. The reddening was found to vary between $E(B-V) \simeq 0.15$ and $0.20 \mathrm{mag}$. All systems were found to be younger than $10 \mathrm{Myr}$, while the field is older than $\sim 50 \mathrm{Myr}$. We also present the luminosity and mass functions of the systems, as well as that of the field. It was found that the luminosity function slope $s$ of the field is steeper than that of the systems, which were found to be $s \simeq 0.12-0.32$. The MF slopes were estimated for both systems and field by directly counting stars between evolutionary tracks. We verify that the MF slopes of the systems are rather shallower than the ones of the field. The MF slopes of the systems lie in the range $-1.0<\Gamma<-2.0$, while those of various fields are significantly steeper, around $\Gamma \simeq-4.0$. LH 95 was found to be probably under disruption. We discuss the possibility that this association is in the process of dissipation.
\end{abstract}

Key words. Magellanic Clouds - stars: emission-line, Be - formation - C-M diagrams - luminosity function, mass function - HII regions - ISM: individual objects: DEM L 251, DEM L 252 - open clusters and associations: individual: LH 91, LH 91-I, LH 95

\section{Introduction}

Giant and super-giant shells are known to be loci of recent star formation (e.g. Chu 1998; Oey 1999). The Large Magellanic Cloud (LMC) is characterized by the large number of such shells (Meaburn 1981; Kim et al. 1999) of which LMC 4 is the most clearly defined. This super-giant shell (SGS) has attracted the interest of various investigations (e.g. de Boer et al. 1998; Efremov \& Elmegreen 1998; Efremov et al. 1998). We present our photometric results of an area on the north-east outer edge of LMC 4, where three stellar associations are located. Our aim is to characterise the stellar populations of the systems and their surrounding field populations.

Westerlund \& Mathewson (1966) noticed a fine HI shell surrounding the whole region of LMC 4, using the

Send offprint requests to: D. Gouliermis,

e-mail: dgoulier@astro.uni-bonn.de survey results of McGee \& Milton (1966a, 1966b). Dopita et al. (1985) have confirmed the existence of the Hi ring with a diameter of $1.8 \mathrm{kpc}$, appreciably larger than the $\mathrm{H} \alpha$ ring $(1.4 \mathrm{kpc})$. From the gas dynamics, they hypothesize that there might be a steady progression of stellar ages, the youngest being found around the periphery. They also calculate that the star-forming region has expanded with a uniform velocity of $36 \mathrm{~km} \mathrm{~s}^{-1}$ over the past $14 \mathrm{Myr}$, sufficient to show stochastic self-propagating star formation (SSPSF). Stellar winds of the massive stars and supernova explosions were considered to be the most important features in LMC 4 dynamics, being adequate to explain its origin and ionization.

More recent results by Braun et al. (1997) and Dolphin \& Hunter (1998) clearly indicate that there is no age gradient within LMC 4, suggesting that a SSPSF scenario cannot explain the formation of the shell. Braun et al. (1997) calculated that $\sim 5-7 \times 10^{3}$ supernovae should have 


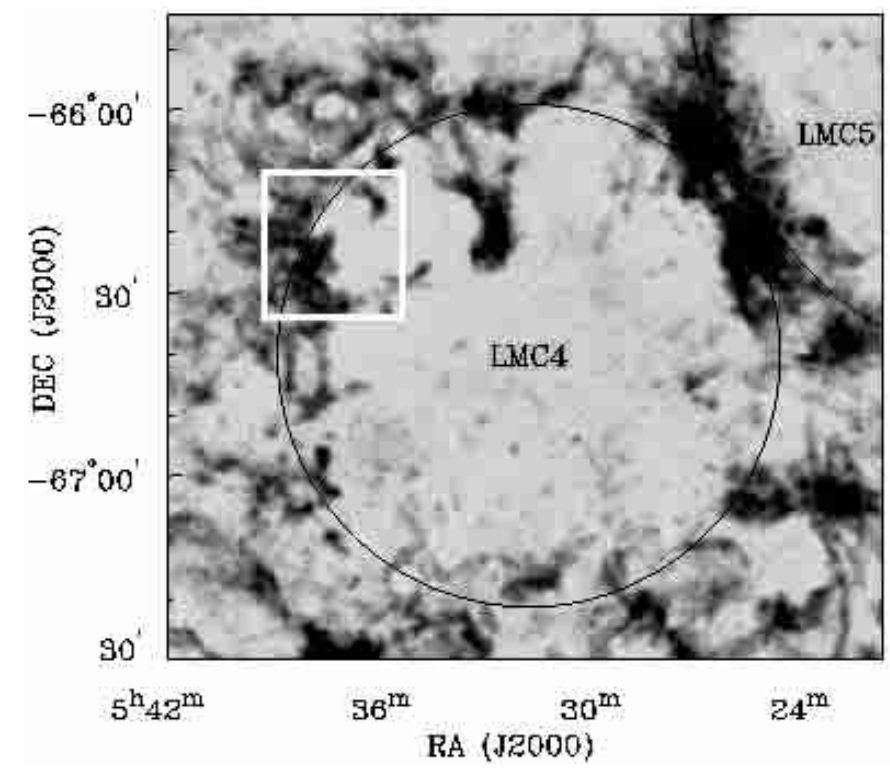

Fig. 1. LMC 4 as shown in the HI map of the LMC by Kim et al. (1997). The region observed in the present study is marked with the white box.

exploded over the last 10 Myr throughout LMC 4 dumping in an area with a diameter of $1.4 \mathrm{kpc}$, rather evenly, the energy disrupting the birth cloud and triggering star formation at the edge. This conclusion is in agreement with the small age span of 9-16 Myr they derived for the stars in a $J$-shaped area inside LMC 4 , and with their most recent result of $10 \pm 2 \mathrm{Myr}$ for the central area of the shell (Braun et al. 2000). They find that these results support the bow-shock scenario for the formation of this SGS presented by de Boer et al. (1998).

The systems we investigate here are among the few visible on the NE side. Figure 1 shows the position of the observed region in relation to LMC 4, as it is observed by Kim et al. (1997) in Hi. LH 91 and LH 95 (Lucke \& Hodge 1970) are two well known stellar associations, still not investigated in any recent work. LH 91 is related to the HiI regions N 64A,B (Henize 1956) or DEM L 251 (Davies et al. 1976), while LH 95 is embedded in the bright HıI region N 64C or DEM L 252.

The best source of optical photometry on these systems, so far, is the one from Lucke (1974), where he presents colour-magnitude diagrams for $96 \mathrm{LH}$ associations. In LH 91 he detected 6 bright MS stars with $13 \lesssim V \lesssim 16$ mag and inside LH 954 OB stars within the same magnitude limits. He also found 3 red stars in LH 91 and none in LH 95. He estimated the foreground reddening of this area to be $E(B-V) \simeq 0.01$, which is the average value for stars near the associations, since, as he noticed, "reddening is highly variable even within a single association".

These systems were also identified as such by Kontizas et al. (1994), who used a method of identifying stellar associations by counting and classifying stars within selected areas of LMC. In this area Kontizas et al. (1994) detected an additional stellar association (LH 91-I), which we include in this investigation. By isochrone fitting on
Lucke (1974) CMDs Kontizas et al. (1994) found an age for both LH $91 \&$ LH $95 \simeq 2 \pm 1 \times 10^{7}$ yr. They counted in $J V R 1.2 \mathrm{~m}$ UK Schmidt photographic plates 16 blue stars in LH 91, 18 in LH 91-I and 15 in LH 95 ${ }^{1}$. They found that these systems are single stellar associations, with densities varying between $0.05-0.08 M_{\odot} \mathrm{pc}^{-3}$, probably belonging to a large aggregate.

Here we analyze $B V R$ and $\mathrm{H} \alpha$ observations of these three systems to better identify and separate them from their field (Sect. 3), to investigate their stellar content, as well as that of the field (Sect. 4) and to construct the corresponding Luminosity and Mass Functions (Sect. 5). In Sect. 6 we analyze the dynamical behavior of the systems and in Sect. 7 we discuss general differences between the systems.

\section{Observations and reductions}

We observed an area of $\simeq 20$ ' $5 \times 20$.' 5 centered on LH 95 $\left(5^{\mathrm{h}} 37^{\mathrm{m}} 1 \mathrm{~s} .7,-66^{\circ} 21^{\prime} 31^{\prime \prime}\right.$ in $\left.\mathrm{J} 2000\right)$. The observations were obtained using direct CCD imaging on the 1-m telescope at Siding Spring Observatory on the night of the 5th of September 1999 under photometric conditions. A Tektronix $2048 \times 2048$ CCD with pixel size equivalent to 0 ". 6 was used for these observations. Typical seeing was $1^{\prime \prime} 5$. The observations were carried out in standard $B V R$ and $\mathrm{H} \alpha$ filters. We took 2 exposures for the $B V R$ filter system $(60 \& 360 \mathrm{~s}$ for $B, 50 \& 300 \mathrm{~s}$ for $V$ and $30 \& 200 \mathrm{~s}$ for $R$ ) and 3 exposures of $600 \mathrm{~s}$ for $\mathrm{H} \alpha$. We averaged the three frames in $\mathrm{H} \alpha$, but they still don't go very deep. The $\mathrm{H} \alpha$ observations show to be adequate for the identification of emission line stars (see Sect. 4.2).

The CCD images were processed with $\mathrm{IRAF}^{2}$ and the photometry of the field was done using the DAOPHOT psf fitting photometry package (Stetson 1987). The standard fields of Landolt (1992) were used to calibrate the photometry in $B$ and $V$. The $R$ and $\mathrm{H} \alpha$ magnitudes were not standardized and the $R-\mathrm{H} \alpha$ colours have an arbitrary zero-point. For the investigation of the systems and their surrounding fields we selected the data, corresponding to an area of $\sim 17 ! 5 \times 17 ! 5$, centred on LH 95. This area selection was based on two criteria: (1) In order to have a very good definition of the field population around each system and (2) so that all the related Hir features be fully apparent in the area. Figure 2 shows the star chart of this area, as well as the corresponding $\mathrm{H} \alpha$ "contour" image.

In order to determine the equatorial coordinates of the stars we used the STScI Guide Star Catalogue (GSC $1.1^{3}$ ),

\footnotetext{
${ }^{1}$ Kontizas et al. (1994) mistakenly switched the LH numbers 91 and 95 in their Table 7.

${ }^{2}$ IRAF is distributed by the National Optical Astronomy Observatories, which is operated by the Association of Universities for Research in Astronomy Inc. (AURA) under cooperative agreement with the National Science Foundation.

3 The Guide Star Catalog was produced at the Space Telescope Science Institute under U.S. Government grant. These data are based on photographic data obtained using the Oschin Schmidt Telescope on Palomar Mountain and the UK Schmidt Telescope.
} 

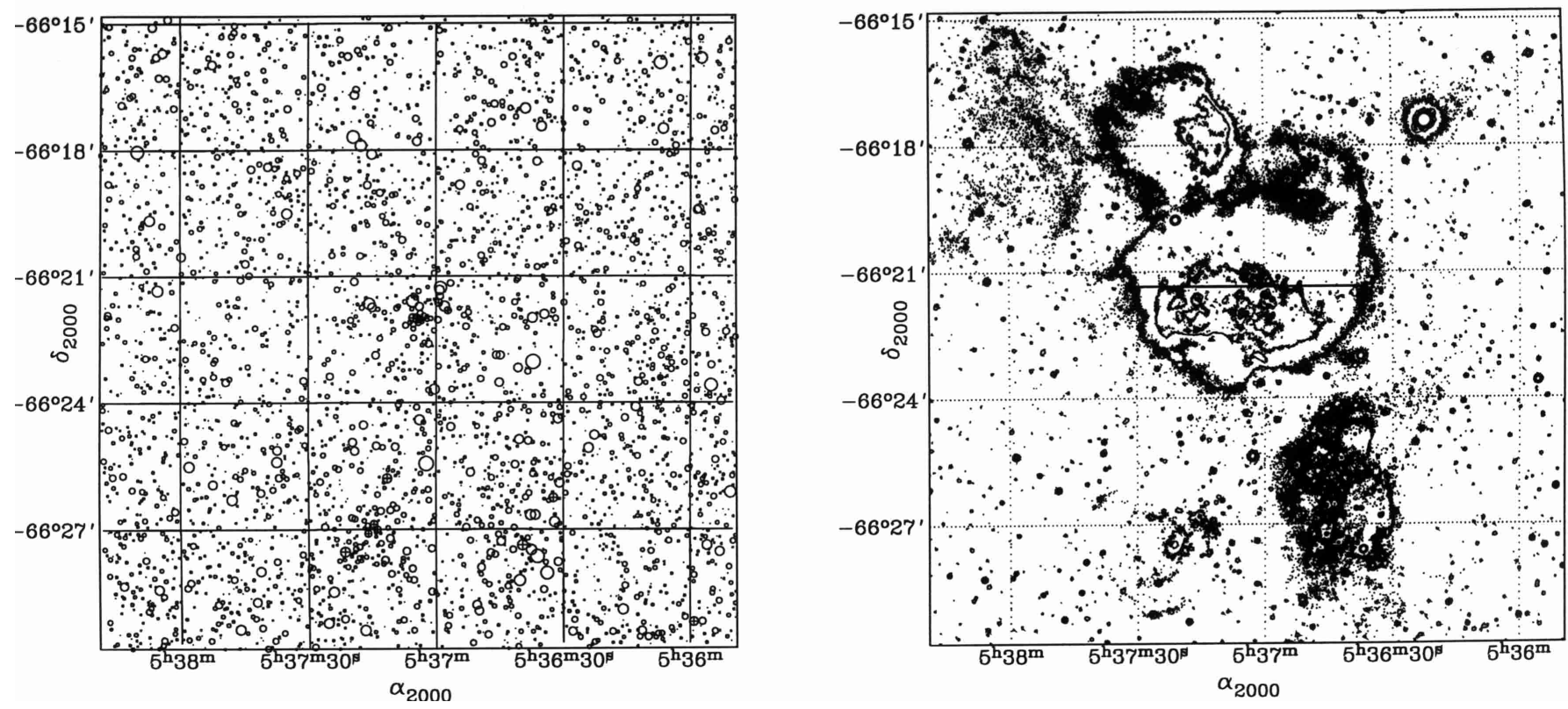

Fig. 2. Positional overview from our data in the field of the systems studied. Left: Star Chart of the selected area down to $V \simeq 22 \mathrm{mag}$. The crossed circle symbols $(\oplus)$ represent the locations of the identified Be stars. Right: the H $\alpha$ image of the area. The lowest contour shown represents signal $2 \sigma$ above the background, where $\sigma$ is the standard deviation of the background. Selected contours are shown, so that the $\mathrm{H} \alpha$ features are more clearly apparent.

available in the Internet. We identified the stars common to our catalogue and the GSC by plotting both data sets in a $x, y$ Cartesian system of coordinates, where $x, y$ are in arcsec. For the whole area of our frames 189 GSC stars were available. We selected fifty five of our stars found also in the GSC. These stars are rather evenly distributed over the frames. We did not use stars found in both catalogues in crowded areas to avoid confusion in the crossidentification. IRAF/CCMAP was used for the transformation of the optical $X, Y$ pixel coordinates of the stars into RA, DEC.

\section{Topography of the systems}

In order to specify the limits of each stellar agglomerate we performed star counts in a grid of $5 \mathrm{pc} \times 5 \mathrm{pc}$ squares. The gray scale isodensity map produced from these counts is shown in Fig. 3. We estimated the background density and the corresponding standard deviation $(\sigma)$ in the whole selected area. Our specification of the extent of each system was based on the $3 \sigma$ isodensity contour line.

In order to determine the density of the background, we performed star counts and estimated the background density and the corresponding standard deviation $(\sigma)$ taking into account the statistics from the whole region, as well as from smaller areas around each association. From the map based on the statistics of the whole region (Fig. 3) and from the contour maps of the smaller areas around each system it was found that while LH 95 \& LH 91-I are well defined by the star counts, LH 91 turns out not to be a well peaked system, which makes the determination of its extent difficult.

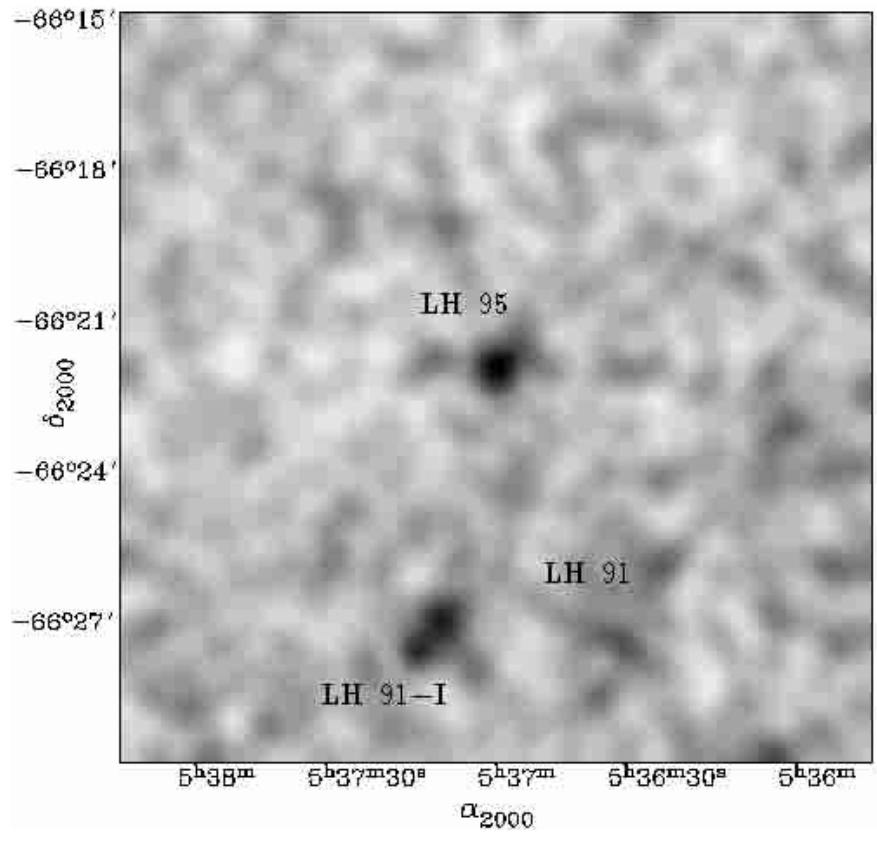

Fig. 3. Gray scale isodensity map produced by counting all stars found in the area. The statistically significant densities, which represent surface stellar density at least $3 \sigma$ above the local background are marked by the darkest gray tones, while the lower density levels are represented by lighter tones. The positions of the three associations are marked.

\subsection{Optical topography}

The limits of each system have been estimated by Lucke \& Hodge (1970), and one can use these results as 

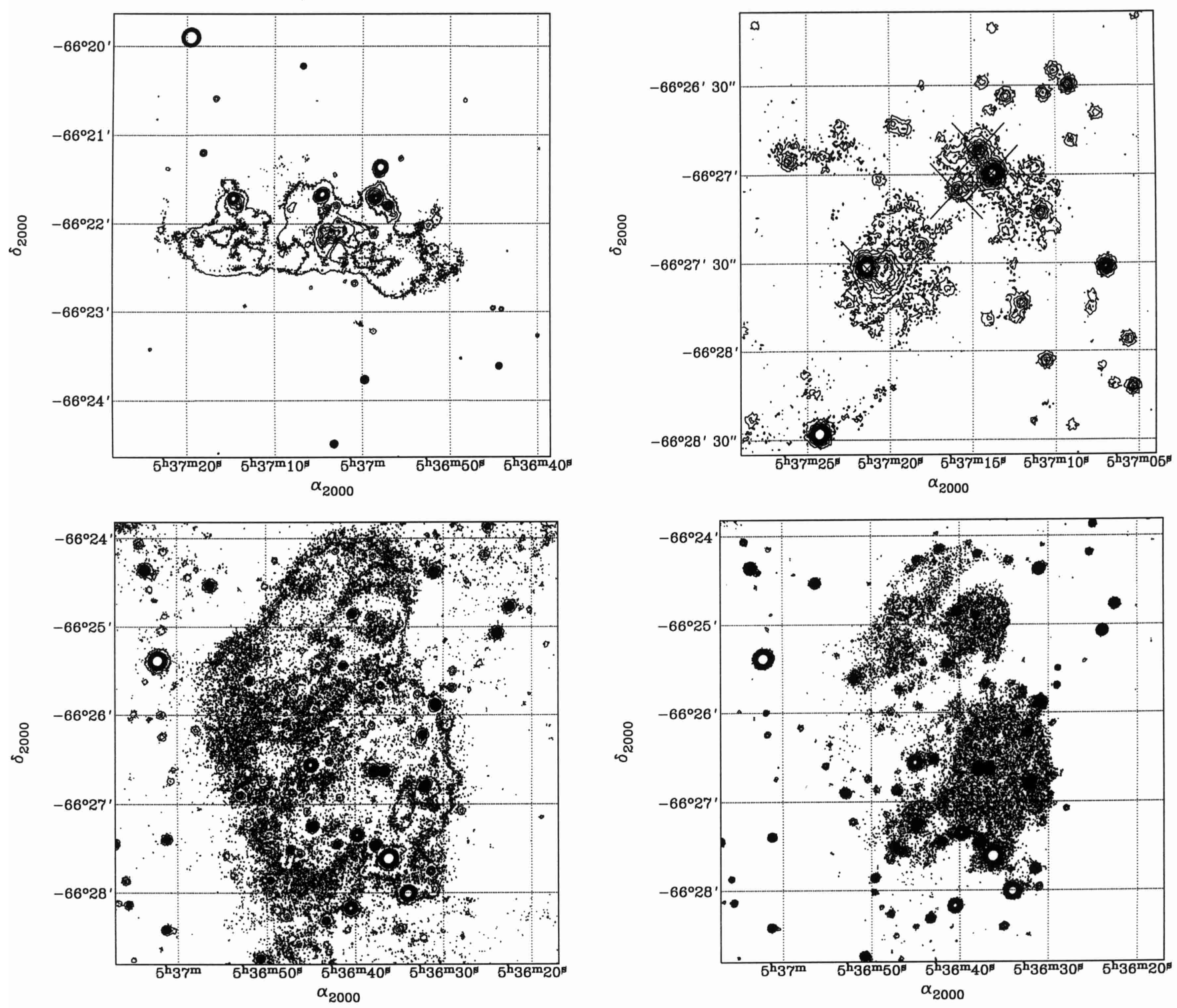

Fig. 4. H $\alpha$ maps. Top panel: left: $\mathrm{H} \alpha$ map of an $5^{\prime} .0 \times 5^{\prime} .0$ area centred on LH 95. Right: H $\alpha$ map of an $2^{\prime} .5 \times 2{ }^{\prime} 5$ area centred on LH91-I. The Be stars found are marked with large $\times$ symbols. Both maps have been plotted for significance $2 \sigma$ above the local background. Bottom panel: $\mathrm{H} \alpha$ map of an 5.0 $\times 55^{\prime} .0$ area on LH 91 for $1 \sigma$ (left) and $2 \sigma$ (right) significance.

representative of each system's extent (e.g. Massey et al. 1995). LH 95 was found by these authors to have size $1.5 \times 1.5$. A more detailed investigation by Kontizas et al. (1994) showed that this association has a size $\simeq 3$ '3. From our star counts it was found that the diameter of LH 95 is little more than about 2 '.0. So we selected a radius for LH 95 of $\simeq 1$ '2 to construct its CMD. LH 91-I was found on the star count contour map to have almost the same size as LH 95. Kontizas et al. (1994) found a size of $\simeq 3$ '4, based also on star counts. In both investigations the size of each system is defined by the boundaries of the corresponding areas within a contour of density $\geq 3 \sigma$.

The systematic differences of our estimations from the ones by Kontizas et al. (1994) can be explained by two factors: (i) They used larger grid element size (11 pc) probably due to the small number statistics on the photographic plates and (ii) they based their estimations on data from photographic plates not only in $V$, but also in $J$ and $R$, while we counted sources found in both our $V$ and $B$ frames. Still the differences in the sizes of the systems are not dramatically large. As far as LH 91 concerns Lucke \& Hodge (1970) found a size of $\simeq 2$ ' $0 \times 2$ ' 0 , while Kontizas et al. (1994) found the maximum dimension of the system to be $\simeq 3{ }^{\prime} 0$. As we show below we selected an area centred on the related HII region (DEM L 251) with diameter of $5 ! 0$ as the most adequate for this system.

\subsection{Ho topography}

Additional information on the topography of the systems can be given from the $\mathrm{H} \alpha$ observations. OB stellar associations are known to be mostly related to HiI emission (e.g. 
Hodge 1986). Indeed it is shown from Fig. 4, where the $\mathrm{H} \alpha$ contour images are given, that all three systems are strongly related to such emission. LH 95, as also visible in the $\mathrm{H} \alpha$ image of the whole area in Fig. 2, is related to a huge HII region of size (as is defined from the $2 \sigma$ contour line $) \simeq 65 \mathrm{pc} \times 85 \mathrm{pc}(4 ! 1 \times 5 ! 3)$. The system shows to be located in the most active "centre" of this region (in terms of intensity). A more detailed discussion on this relation is given in Sect. 7. On the other hand the HII emission in the region of LH 91-I shows to be very diffuse, not showing any particular feature and is directly related to the identified emission stars (Fig. 4 - top right panel).

The lower panel of Fig. 4 is dedicated to LH 91, where we show the $\mathrm{H} \alpha$ image for $1 \sigma$ (bottom left panel) and $2 \sigma$ significance (bottom right panel) correspondingly. What is shown from these maps is that indeed the area of LH 91 is related to an HII region, which appears to be large (with maximum size $\simeq 5$ ! 0 in the $1 \sigma$ map) but rather diffuse (since it is apparent only in the $1 \sigma \mathrm{H} \alpha$ map, while in the $2 \sigma$ map one can see only HiI patches). It is interesting here to note that LH 91 as far as the star counts are concerned does not seem to represent a stellar system in the "classical" meaning (concentration of stars physically related to each other). This is in line with Lucke (1974), who states that LH 91 may not be an association at all. So, in the case of the area around LH 91, we will base ourselves on the $\mathrm{H} \alpha$ maps to select the area, in which we will examine the stellar population. This area has dimensions $\simeq 48 \mathrm{pc} \times$ $80 \mathrm{pc}\left(3^{\prime} 0 \times 5{ }^{\prime} \cdot 0\right)$. Thus we selected it to have a maximum size of $\simeq 5$ '0.

\section{Colour-magnitude diagrams}

\subsection{Radial colour-magnitude diagrams}

We produced the colour-magnitude diagram (CMD) $V$ (vs.) $B-V$ for various radial distances from the geometrical centre of each association. These are shown in Fig. 6 for LH 95 and LH 91-I and in Fig. 7 for the area of LH 91. In Fig. 5 are shown the selected areas of radius 2.5 each around the systems, as well as the four most "empty" areas of our frame (empty fields), which we used for the investigation of the background population of the observed area. In this paper we will use "background field" to mean the sum of the empty fields shown in Fig. 5 (Field 1, Field 2, Field 3, Field 4). For the cases of the areas around LH 91-I and LH 95, outside the specified limits of the systems up to radial distance $2 \cdot 5$, we will use the term "surrounding field".

As shown in Fig. 6 from the radial CMDs of LH 95, main-sequence stars with magnitudes between $V \simeq 19$ 16 mag firstly appeared within 0.4 arcmin from the centre, while main-sequence populations fainter than $V=19 \mathrm{mag}$ and brighter than $V=16$ mag start to appear outside this distance, where also some red stars can be seen. The most blue and bright stars are located within the system's limits (in a radius of $\sim 1 ! 2$ ), while outside these limits the stellar population is characterized by redder colours and

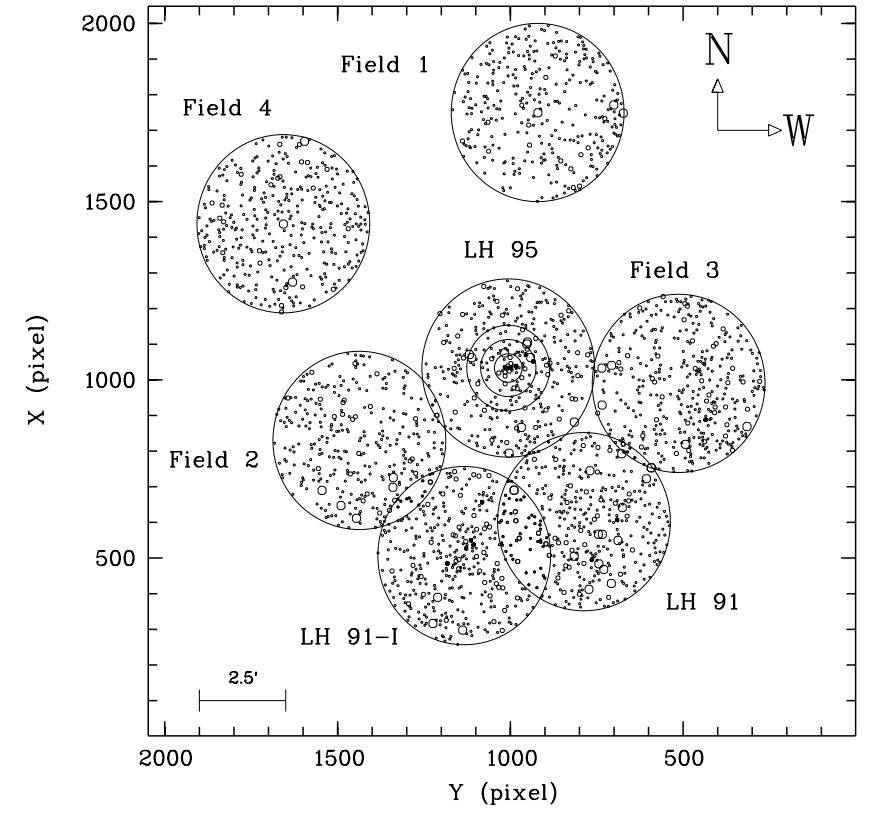

Fig. 5. Map of the areas selected for the study of the stellar populations of the systems, their surrounding fields and the background field. The background field is defined as the sum of Field 1 to Field 4, serving as reference background. In the case of LH 95 the annuli used for the derivation of the CMD at various radial distances have been overplotted. Each selected area has a radius of 2.5 .

fainter magnitudes. So, the radial limit of the younger population shows to agree with the limits of the system as were selected from the star count contour maps.

In the case of LH 91-I, while the system was found more or less to have the same size as LH 95, an upper MS population is still apparent outside the radial distance of $\sim 1$ '.2. More specifically, by examining the CMD around LH 91-I for radial distances between 1'.2 and 2'.5, one can see that there are two brighter MS stars outside the limits of the system, one of them being a Be star. The well defined MS of the surrounding field population, seems to suggest that LH 91-I is embedded in a younger field than the one of LH 95.

We produced the radial CMDs for the region around LH 91, choosing as a centre the geometrical centre of the corresponding HiI region. As is shown from Fig. 7 the younger population is concentrated within a radius of $\sim 2$ ' 0 . Lower MS stars are apparent outside this limit, where the red clump is also well defined. For the estimation of the age of this area (Sect. 4.4) we will use the stellar population in the whole region defined by a radial distance of $2 ! 5$, since as we already stated we couldn't identify any significant stellar concentration toward LH 91.

\subsection{Identification of Be stars}

Photometric methods for the identification of Be stars have been presented by Grebel et al. (1992), Grebel (1997) and Keller et al. (1999). We have identified the $\mathrm{H} \alpha$ emission stars, following these methods, by plotting the colour 

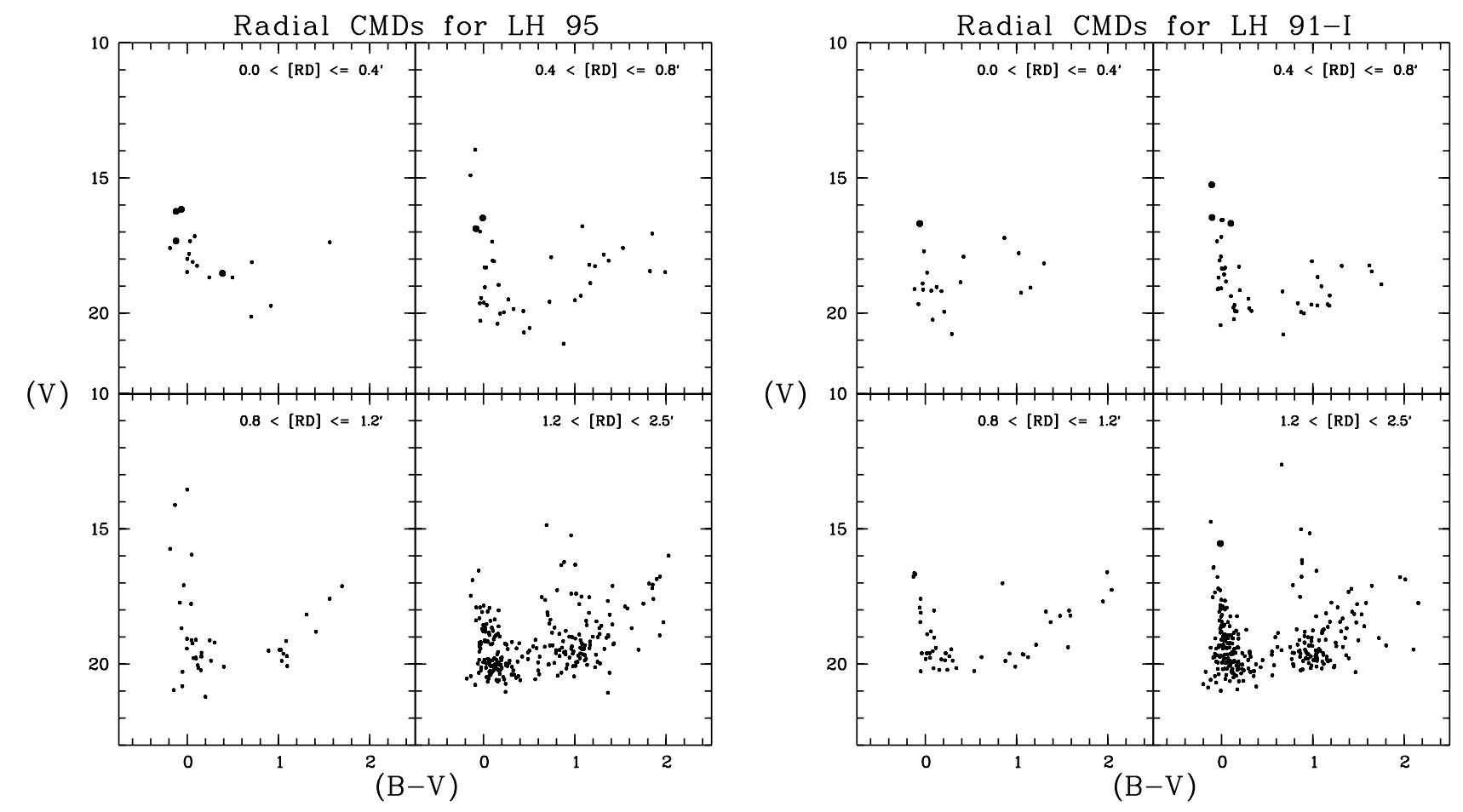

Fig. 6. CMDs for annuli of indicated size around the centre of LH 95 \& LH 91-I. Thick points represent the Be stars.

Table 1. Characteristics of the Be stars identified in the whole observed region. Coordinates are given in J2000.

\begin{tabular}{|c|c|c|c|c|c|c|}
\hline \multirow[t]{2}{*}{ ID } & \multirow[t]{2}{*}{ System } & $\mathrm{RA}$ & Dec & \multirow[t]{2}{*}{$\bar{V}$} & \multirow[t]{2}{*}{$B-V$} & \multirow[t]{2}{*}{ Note } \\
\hline & & $\mathrm{h} \quad \mathrm{m} \quad \mathrm{s}$ & 0,11 & & & \\
\hline$\overline{\overline{12}}$ & 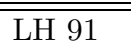 & 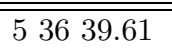 & -662722.57 & 14.85 & $=0.053$ & \\
\hline 37 & LH 91-I & 53721.37 & -662731.82 & 15.25 & -0.107 & $\dagger$ \\
\hline 54 & & 53711.51 & -662547.40 & 15.54 & -0.013 & \\
\hline 70 & & 53559.07 & -66 2912.04 & 15.72 & -0.071 & \\
\hline 88 & LH 91 & 53632.33 & -66 2614.73 & 15.98 & -0.084 & \\
\hline 114 & LH 95 & 53705.38 & -66 2159.13 & 16.16 & -0.064 & \\
\hline 124 & LH 95 & 53704.38 & -662200.53 & 16.24 & -0.122 & \\
\hline 154 & LH 91-I & 53714.62 & -662652.05 & 16.46 & -0.105 & \\
\hline 157 & LH 95 & 53659.33 & -662137.54 & 16.48 & -0.009 & \\
\hline 158 & & 53808.71 & -663132.61 & 16.44 & -0.070 & $\dagger$ \\
\hline 193 & LH 91-I & 53715.95 & -662705.59 & 16.69 & -0.061 & $\dagger$ \\
\hline 194 & LH 91-I & 53713.83 & -662659.60 & 16.68 & 0.101 & \\
\hline 203 & & 53838.76 & -662325.18 & 16.75 & 0.015 & $\dagger$ \\
\hline 218 & & 53525.15 & -662333.28 & 16.80 & -0.033 & $\dagger$ \\
\hline 239 & LH 95 & 53657.14 & -662148.53 & 16.87 & -0.084 & \\
\hline 266 & & 53621.43 & -663109.79 & 16.97 & -0.036 & $\dagger$ \\
\hline 410 & LH 95 & 53703.57 & -662200.03 & 17.33 & -0.123 & \\
\hline 622 & & 53653.99 & -66 3004.48 & 17.75 & 0.010 & \\
\hline 662 & & 53607.06 & -662324.11 & 17.81 & -0.091 & \\
\hline 1066 & & 53606.21 & -662450.32 & 18.27 & -0.031 & $\dagger$ \\
\hline 1388 & LH 95 & 53702.08 & -66 2157.29 & 18.53 & 0.386 & $\star$ \\
\hline $\begin{array}{l}\text { tanc } \\
\text { tanc }\end{array}$ & $\begin{array}{l}\text { star } \\
\text { ine }\end{array}$ & $\begin{array}{l}\text { found a } \\
\text { el to th } \\
\text { mag. }\end{array}$ & ding to th & प1 & $\begin{array}{l}\text { Greb } \\
\text { line }\end{array}$ & $\overline{99}$ \\
\hline
\end{tabular}




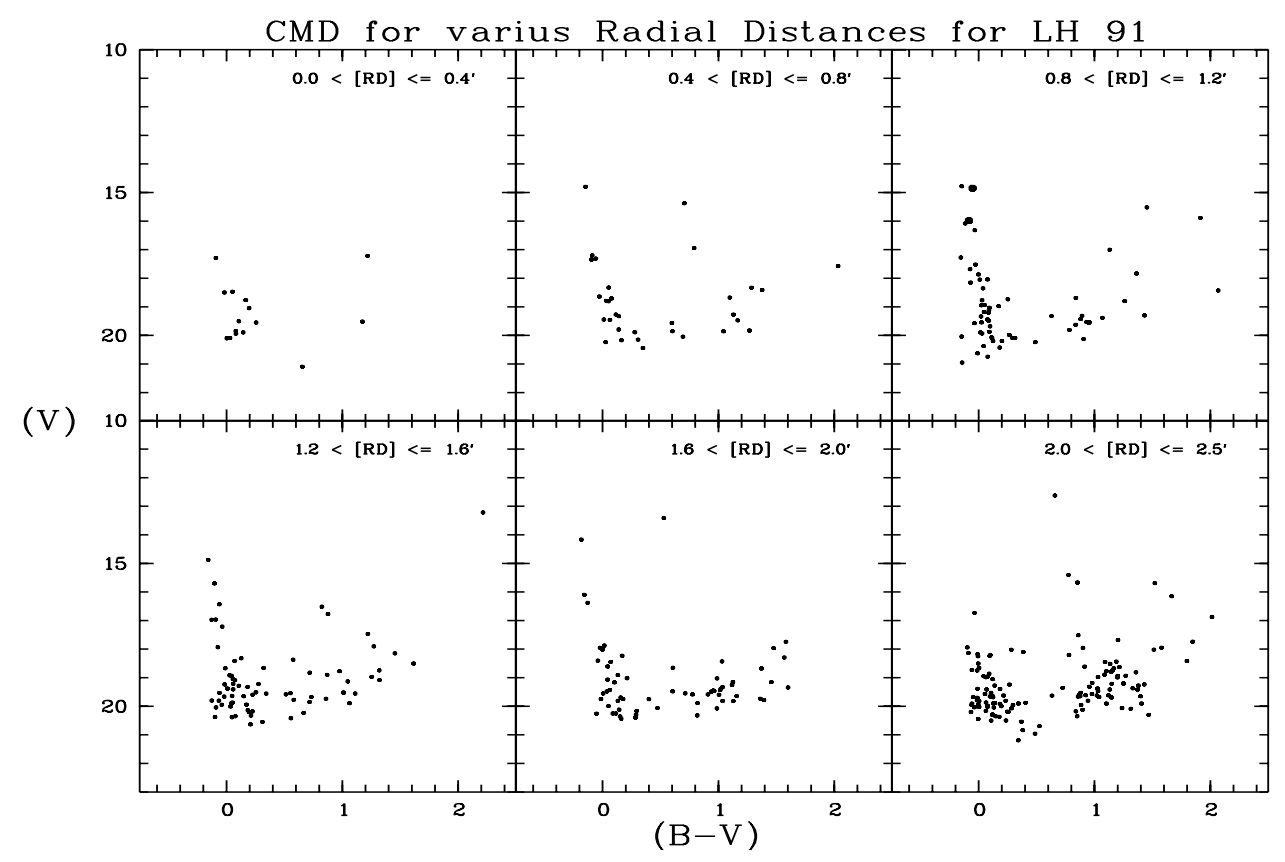

Fig. 7. CMDs for annuli of indicated size around the centre of LH 91. Thick points represent the Be stars.

Table 2. Density of stars in six areas on the CMDs for the systems (inside the selected limits), for the systems surounding fields (outside their limits) and for 4 selected empty fields, as well as for the general Background Field. The numbers are normalized to $1 \operatorname{arcmin}^{2}$ and completeness corrected. The counting uncertainties are also given. The difference between "system" and "field" in parenthesis for LH 95 and LH 91-I is that "system" is used for the area within radial distance $r<1$ '.2 and "field" for the surounding fields of the systems (for distances $1.2<r<2$ '. 5 ).

\begin{tabular}{l|ccc|ccc}
\hline & \multicolumn{3}{|c}{$13 \leq V<17$} \\
& $B-V<0.6$ & $0.6<B-V<1.3$ & $1.3<B-V$ & $B-V<0.6$ & $0.6<B-V<1.3$ & $1.3<B-V$ \\
\hline \hline LH 95 (system) & $2.67 \pm 0.77$ & $0.23 \pm 0.23$ & 0.00 & $19.34 \pm 2.07$ & $6.26 \pm 1.18$ \\
LH 95 (field) & $0.14 \pm 0.09$ & $0.33 \pm 0.15$ & $0.20 \pm 0.12$ & $12.41 \pm 0.91$ & $7.32 \pm 0.70$ & $1.97 \pm 0.81$ \\
LH 91-I (system) & $2.05 \pm 0.67$ & 0.00 & $0.23 \pm 0.23$ & $22.32 \pm 2.22$ & $9.32 \pm 1.44$ & $3.21 \pm 0.84$ \\
LH 91-I (field) & $0.33 \pm 0.15$ & $0.40 \pm 0.16$ & $0.14 \pm 0.09$ & $14.48 \pm 0.98$ & $7.33 \pm 0.70$ & $1.79 \pm 0.34$ \\
LH 91 & $0.82 \pm 0.20$ & $0.31 \pm 0.13$ & $0.31 \pm 0.12$ & $14.84 \pm 0.87$ & $7.11 \pm 0.60$ & $1.91 \pm 0.31$ \\
Field 1 & $0.05 \pm 0.05$ & $0.21 \pm 0.10$ & $0.05 \pm 0.05$ & $10.34 \pm 0.73$ & $9.56 \pm 0.70$ & $1.33 \pm 0.26$ \\
Field 2 & $0.26 \pm 0.11$ & $0.16 \pm 0.09$ & $0.15 \pm 0.09$ & $9.69 \pm 0.70$ & $9.26 \pm 0.69$ & $2.06 \pm 0.32$ \\
Field 3 & $0.25 \pm 0.11$ & $0.10 \pm 0.07$ & $0.21 \pm 0.10$ & $14.79 \pm 0.87$ & $9.73 \pm 0.70$ & $1.48 \pm 0.27$ \\
Field 4 & $0.10 \pm 0.07$ & $0.21 \pm 0.10$ & $0.21 \pm 0.10$ & $11.32 \pm 0.76$ & $11.39 \pm 0.76$ & $1.31 \pm 0.26$ \\
Background Field & $0.17 \pm 0.05$ & $0.17 \pm 0.05$ & $0.16 \pm 0.04$ & $11.53 \pm 0.38$ & $9.98 \pm 0.36$ & $1.55 \pm 0.14$ \\
Foreground & $\lesssim 0.13$ & $\gtrsim 0.09$ & 0.02 & $\lesssim 0.28$ & $\gtrsim 0.29$ & 0.50 \\
\hline \hline
\end{tabular}

index $R-\mathrm{H} \alpha$ against the colour index $B-V$, as shown in Fig. 8.

In order to select our sample of Be stars we plot a line parallel to the sequence of non-emission line stars. The distance of this line from this sequence depends on the selection criterion. We have drawn this line for distance $0.4 \mathrm{mag}$ (following Keller et al. 1999) and $0.2 \mathrm{mag}$ (following Grebel 1997). The characteristics of the Be stars identified are shown in Table 1.

\subsection{Field population}

We selected four fields possessing minimal structure in the isodensity map (Fig. 3) to investigate the background population. These are shown in Fig. 5 (Field 1 to Field 4). We present the overall CMD of these areas (background field) in Fig. 9 (bottom - right panel). One can see that in the background field most of the population consists of stars on the lower end of the observed main-sequence, as well as of red clump and red giant branch stars. These features can also be seen in the case of LH 91 and faintly in the CMDs of LH 95 and LH 91-I (Fig. 9). These results are in agreement with the result of Kontizas et al. (1994), that the systems do not seem to be embedded in a general field of OB stars. Indeed the CMDs for radial distances further than 1'.2 from the centre of LH 95 and 2'.0 from the centre of LH 91 (Figs. 6 and 7) show features that seem to coincide with the ones of the background field CMD, while 


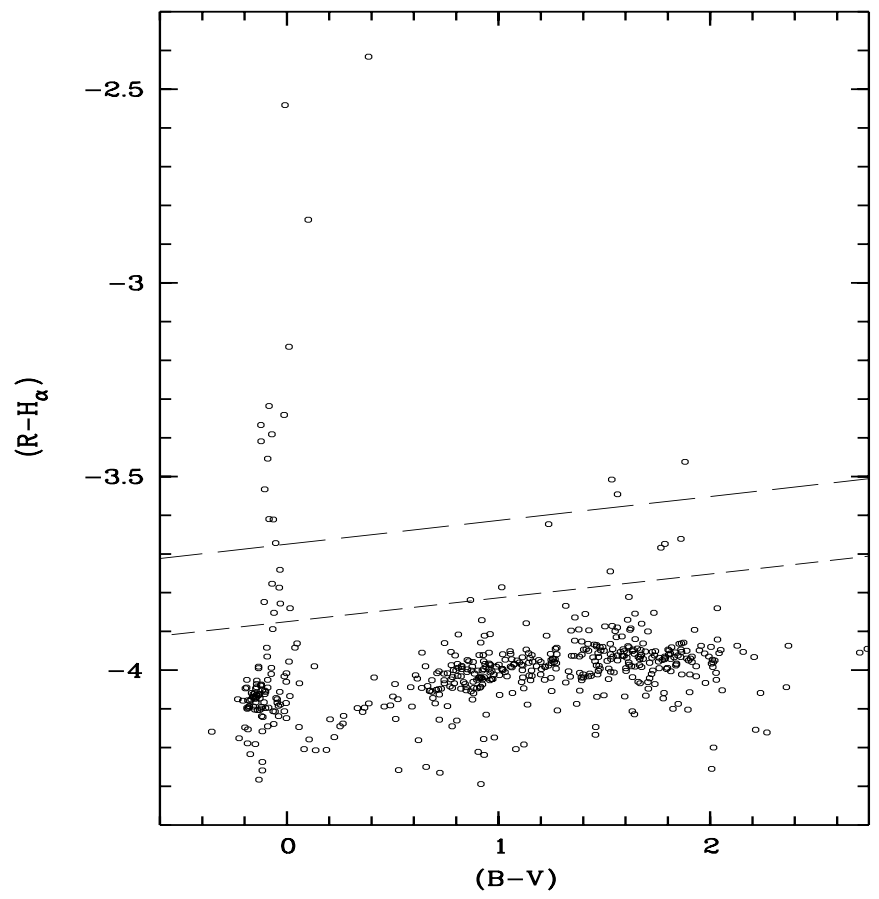

Fig. 8. Be star diagnostic diagram. Most of the main-sequence stars lie in a clump around $R-\mathrm{H} \alpha=-4.07$ and $B-V=$ -0.22 . The spread of normal MS stars in the $R-\mathrm{H} \alpha$ direction about this point is mostly due to the photometric errors in the narrow-band $\mathrm{H} \alpha$ magnitudes. A second group of cooler stars form an almost horizontal band. The third group of stars extending up and to the red of the MS clump represents the stars with significant $\mathrm{H} \alpha$ emission and they are Be stars. Some red giant stars exhibit also strong $\mathrm{H} \alpha$ emission, but these are located to the cooler colours.

the surrounding field of LH 91-I is well populated by MS stars with magnitudes 14 mag $\lesssim V \lesssim 18$ mag. In addition in the background field CMD there are few bright MS stars with magnitudes $V<16$ mag. The brighter background field MS star $(V \lesssim 14.0 \mathrm{mag}$ ) is located in Field 4 (Fig. 5) and does not show to belong in any particular system.

In order to have more quantitative information on the differences of the stellar populations between the systems and the various fields we divided every CMD in six colour and brightness regions and we counted the stars in each one of them (see Table 2). The analysis shows that the number of background field MS stars brighter than $V=$ 17 mag in the CMDs of the systems is less than $15 \%$ for both LH 95 and LH 91-I. The area of LH 91 is also seen to include a much higher number of upper MS stars than the background field. On the other hand the background field includes an adequate number of lower MS stars (with $V \geq$ $17 \mathrm{mag}$ ), which represent a fraction of about 50 to $80 \%$ in the systems' CMDs for stars with $(B-V)<0.6$ mag. It is interesting to note that the faint populations of $\mathrm{LH} 95$ and LH 91-I with $0.6<(B-V)<1.3 \mathrm{mag}$ show to be completely dominated by field stars, while field stars represent a fraction no more than $50 \%$ of the systems' stars with $(B-V)>1.3 \mathrm{mag}$. There are also some galactic foreground stars expected in our CMDs, the numbers of which are shown in the last row of Table 2, as were estimated by
Ratnatunga \& Bahcall (1985). The contribution of these stars in most of the systems' CMD areas is very small, while it seems that they account for a significant fraction of field stars with $13 \leq V<17 \mathrm{mag}$ and $B-V<1.3 \mathrm{mag}$.

\subsection{Age of the systems and of the background field}

The determination of the age of the systems was made by using isochrone models for $Z=0.008$, the proper metallicity for young LMC populations like stellar associations (e.g. Westerlund 1997). We used the same models for the estimation of the age of the background field. We assumed a distance modulus $m-M=18.55$ mag as representative of previous investigations (e.g. Alcock et al. 1996; Caputo 1997). We used isochrone models from the Geneva (Schaerer et al. 1993), as well as from the Padua (Alongi et al. 1993) group. These models differ somewhat in their treatment of convection, core overshoot and mass loss. From our isochrone fittings it was found that the two models do not show significant differences in the representation of the evolution of our systems (the difference found to be of the order of $\log ($ age $) \simeq 0.1)$. As a consequence we present the ages based only on the Geneva models.

\subsubsection{Reddening toward the systems}

For early-type stars, the reddening free Wesenheit function can be used for the estimation of colour excesses (Madore 1982):

$$
\begin{aligned}
W & =V-R_{V}(B-V) \\
& =V_{0}+A_{V}-R_{V}(B-V)_{0}-R_{V} E(B-V) \\
& =V_{0}-R_{V}(B-V)_{0} .
\end{aligned}
$$

The intrinsic colour $(B-V)_{0}$ is a unique function of the $M_{W}$ magnitude along the Zero Age Main Sequence (ZAMS), so it can be used for the estimation of the colour excess, $E(B-V)$, of MS stars only. There are, though, two constraints that must be considered. The $M_{W}$ magnitude is distance dependent, so it must be corrected for the distance of the stars with the use of the appropriate distance modulus. Here we assume the same adopted distance modulus for all stars observed. Secondly, in order to obtain the correct values of the colour excesses, one should select the stars that are as close as possible to the ZAMS, meaning the unevolved MS stars.

Many studies (starting with Olson 1975 and Turner 1976) have been made to establish an accurate value of the ratio $R_{V}$ (reddening curve) of the total absorption in $V\left(A_{V}\right)$ to the colour excess $(E(B-V))$ :

$A_{V}=R_{V} E(B-V)$.

A generally accepted value of $R_{V}$ is $R_{V}=3.2$ (Mihalas \& Binney 1981). This value is comparable with that found by Koornneef (1983: $\left.R_{V}=3.1\right)$. Leitherer \& Wolf (1984) found a similar empirical reddening curve $\left(R_{V} \simeq 3.13\right)$. In this investigation we will adopt the value $R_{V}=3.1$

We choose magnitude and colour limits for the MS population for the associations, by plotting isochrone 

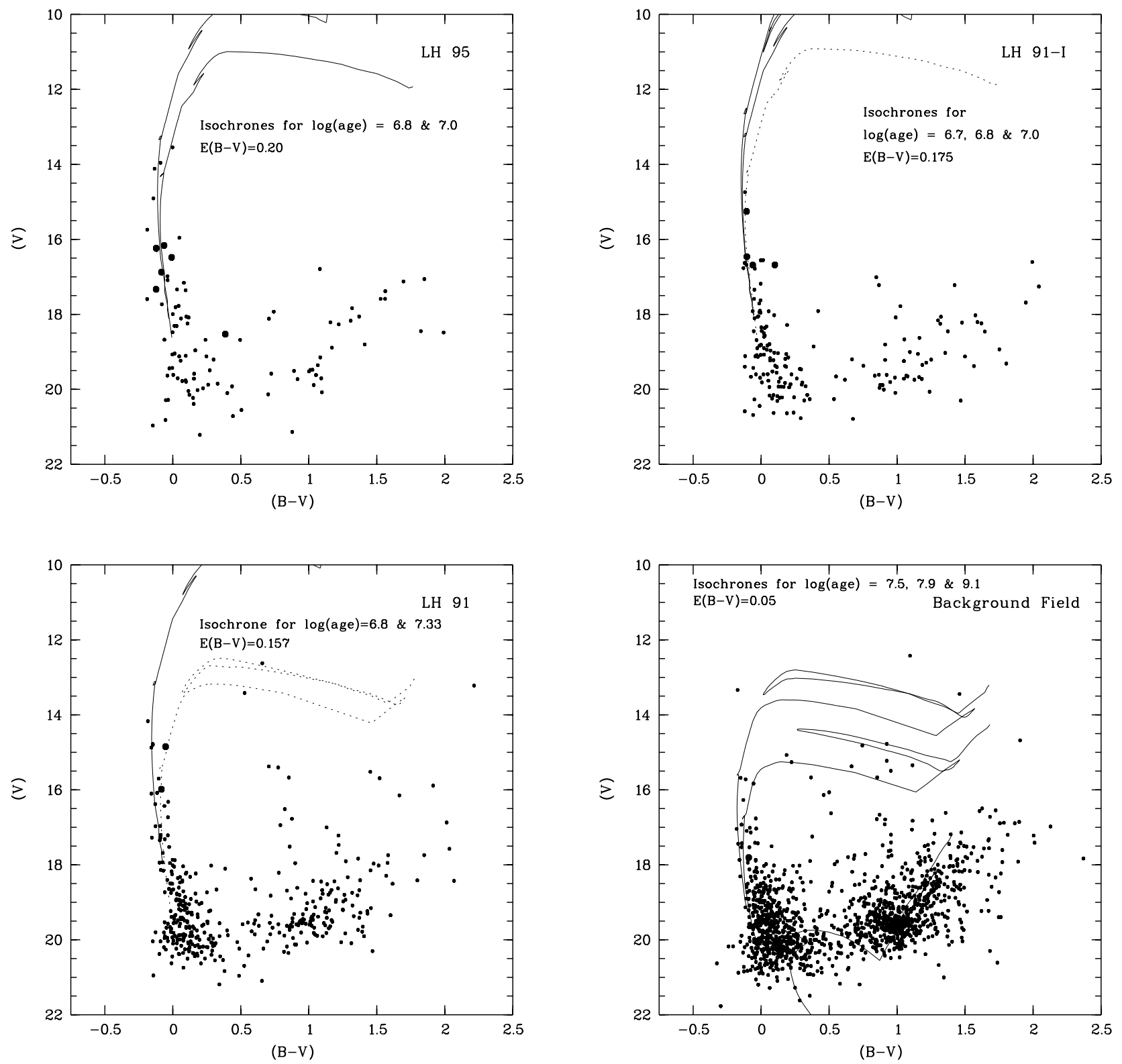

Fig. 9. Top panel: CMDs for LH 95 and for LH 91-I (for stars with radial distances $\leq 1.2$ from the centre in both systems). Bottom panel: CMDs for LH 91 (for stars with radial distances $\leq 2.5$ from the centre) and for the background field. In all cases thick circles represent the Be stars. The corresponding isochrones have been overplotted.

models on each CMD. In the case of LH 91 and LH 91-I these limits were chosen to be $V \leq 19.0$ mag and $B-V \leq$ $0.0 \mathrm{mag}$, while for LH 95 we selected stars brighter than $V \simeq 18.0$ mag with colours $B-V \leq 0.1$ mag. These limits show to be in line with the ones used by Hill et al. (1994) for the estimation of the interstellar reddening toward 14 OB stellar associations in the Magellanic Clouds by applying the Wesenheit function. The distributions of the colour excesses for the selected stars give a mean colour excess $E(B-V)=0.1-0.2 \mathrm{mag}$ for all systems. The most obscured areas are the ones of LH $95(E(B-V)=0.20 \pm$ $0.08)$ and of LH 91-I $(E(B-V)=0.18 \pm 0.04)$, while LH 91 has $E(B-V)=0.16 \pm 0.04$. These reddenings are in line with the value found by Will et al. (1996), $E(B-V)=0.20$ in the area of NGC 1948 on the north-west edge of LMC 4.
For the age estimation these values were not accepted a priori. We fitted several isochrones on the CMDs by eye and verified that the estimations of the reddening as given above are correct. The foreground reddening for this region of LMC as found from the map of Oestreicher et al. (1995) is around $E(B-V)=0.05$ mag. Thus the major fraction of the reddening toward our systems is due to LMC gas. This suggests that differential reddening within the area investigated should be expected.

\subsubsection{Age estimation}

In Fig. 9 we show the selected CMDs of the systems and of the background field with the most appropriate 


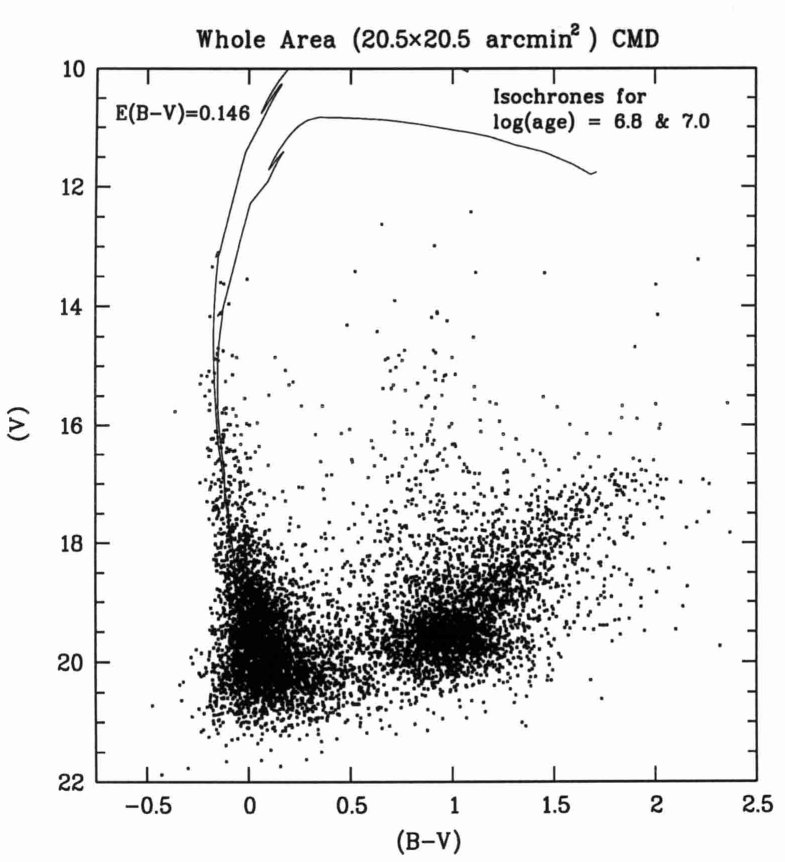

Fig. 10. CMD of the whole observed area, with isochrone models overplotted.

isochrone models overplotted for distance modulus $m-$ $M=18.55 \mathrm{mag}$. The classical method of isochrone model fitting on the observed CMD of young systems cannot be easily applied, due to the fitting difficulties for the upper main sequence. Thus we selected the isochrones that represent the upper age limit of our systems, as shown in Fig. 9. All systems are very young with ages no older than $\simeq 10$ Myr. In the case of LH 95 ages as young as $\sim 8 \mathrm{Myr}$ can be assigned, while for LH 91-I a young age seems to be applicable, but with a large uncertainty due to the lack of MS stars brighter than $V \simeq 14.5$ mag. Taking into account, though, the brightness of the brightest blue star in the CMD the age of LH 91-I could be estimated at about 10 Myr.

The background field (the sum of all four empty fields) seems to be much older with an age not younger than $\sim 50 \mathrm{Myr}$ as shown by the corresponding isochrones fitted. A red clump-branch population well fitted by an $\sim 1.25$ Gyr isochrone is also apparent. Given the presence of stars between the isochrones of $50 \mathrm{Myr}$ and 1.25 Gyr, stars several Myr older than 50 Myr are present, suggesting that the background field is not neccesarily representing a single-age population. For the background field age estimation we used reddening of $E(B-V)=0.05$. It is interesting to note that the age of the systems is similar to the youngest age seen in the whole observed region. We present the CMD of this region in Fig. 10 with the Geneva isochrones for log (age) 6.8 and 7.0 (6.3 and $10 \mathrm{Myr}$ ) overplotted. There are some red stars with magnitudes $13 \lesssim V \lesssim 17 \mathrm{mag}$ around $(B-V) \simeq 1.0 \mathrm{mag}$, which are most probably Galactic field stars. Indeed in the CMD of the whole region the stellar density for $13 \leq V<17 \mathrm{mag}$ and $0.8<B-V<1.3 \mathrm{mag}$ was

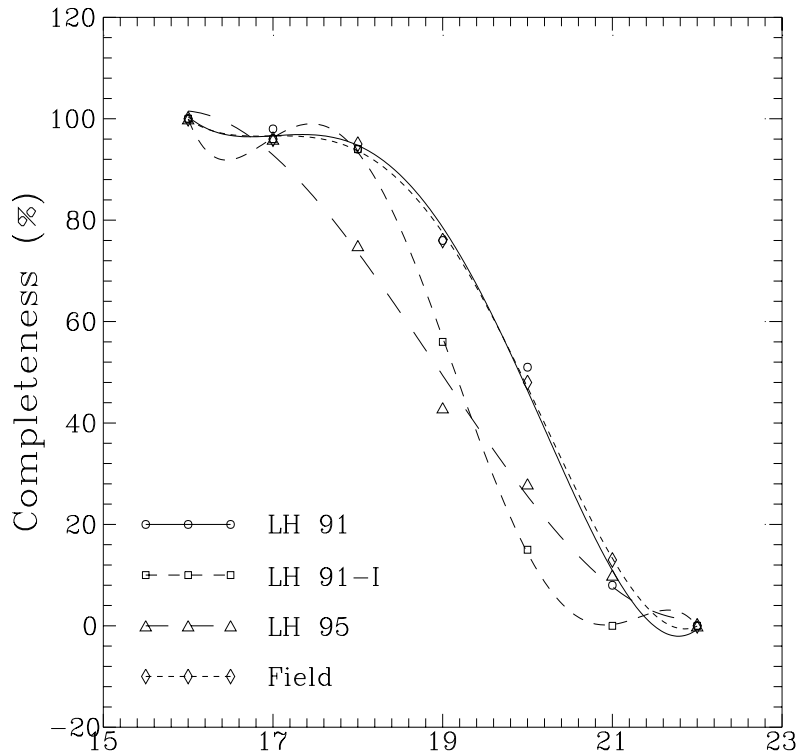

(B)

Fig. 11. Completeness for the areas of the systems and the background field, in terms of percentage of test stars that were added to the frames and recovered. There is a difference in the completeness for the small areas (radius 1.2) around LH 91-I \& LH 95. On the other hand the completeness seems to be almost the same between the background field and the area around LH 91.

found to be $0.06 \mathrm{stars} / \operatorname{arcmin}^{2}$, while the corresponding expected foreground stellar density is higher (Table 2). There are also a few red supergiants.

The reddening used for the isochrone fit on the CMD of the whole observed region is $E(B-V) \simeq 0.15 \mathrm{mag}$, which is the mean value of the reddenings used for the systems and the background field. The isochrones that are placed over the CMD of the whole area treat the stars at $V=13-14$ mag as MS stars. Still, they could be A-type supergiants, since the $B-V$ colours of A supergiants are not that different from the upper MS stars. In order to test this possibility we tried to fit the MS up to $V=14.7 \mathrm{mag}$ with older isochrones and various reddenings, but we couldn't find any model suitable in case these stars were AI stars. Still, one should keep in mind that theoretical isochrones might be uncertain at predicting the positions of the blue loops.

\section{Luminosity functions and mass functions}

Before we proceed to the construction of the LFs we performed several tests for the estimation of the completeness of our photometry. These tests were performed by adding artificial stars of known luminosity into the original frames and completely regenerating the photometry on all stars (true and artificial). The completeness is defined as the number of recovered artificial stars versus the total number added in each frame. The tests were performed in $B$ since these magnitudes place the limit on the photometry. We checked the completeness of our photometry in 


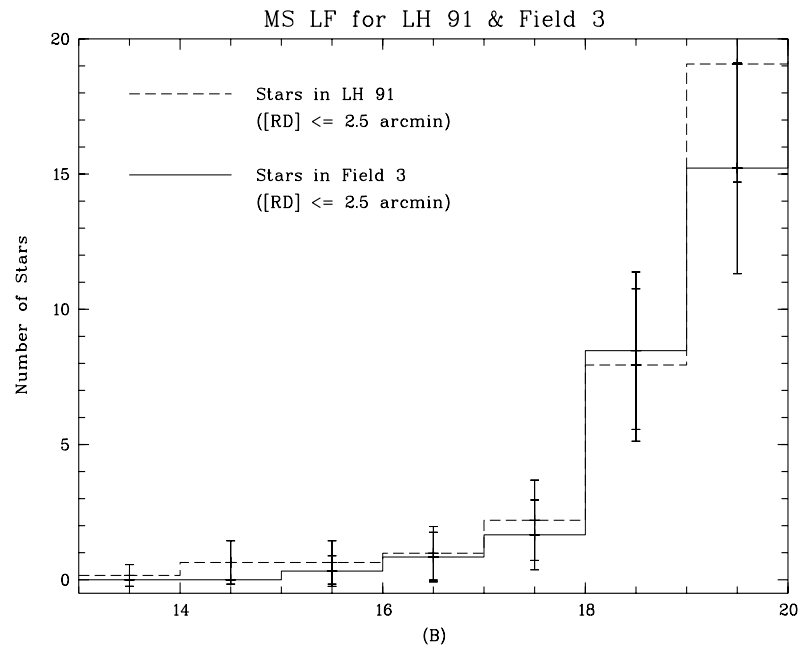

Fig. 12. Differential LF corrected for incompleteness for main sequence stars in LH 91 (dashed line). The completeness corrected MS LF of field 3 is overplotted with solid line.

the areas of LH 91-I \& LH 95 (within radial distance of 1.2 from each centre) and of LH 91 (within 2.5 from the corresponding centre).

We also checked the completeness in the fields surrounding LH 91-I \& LH 95 and in the background field. We found that the completeness within the fields and in the area of LH 91 is not different from the one of the general field. On the other hand we found different completeness functions for the areas of LH 91-I \& LH 95. In Fig. 11 we present our results for the areas of the systems and of the general field in percentage of recovered artificial stars.

\subsection{Luminosity functions}

We constructed the LFs of the systems by binning the observed stars according to their $B$ magnitudes. We selected stars with $B-V \leq 0.5 \mathrm{mag}$ as the most representative of the main-sequence population. In order to check the possibility of biased construction of the LF due to the bin size selection, we performed binning of the stars using different bin sizes. Then we tested the slopes $s$ of the LFs produced $\left(\log N \propto s \cdot B_{\text {bin }}\right)$ for differences. It was found that there is no significant change in the LF slope for bin sizes between 0.5 and $1.5 \mathrm{mag}$. We have binned the stellar luminosities in intervals of $1 \mathrm{mag}$ for all cases.

In Fig. 12 we present the LF corrected for incompleteness derived for the area of LH 91 (dashed line). In order to compare this LF with one derived for an empty field, we also present in the same figure overplotted (with solid line) the LF of Field No. 3, which is the closest field region to LH 91. Both LFs are normalized to the same area. There is no significant difference between the two LFs. This is also demonstrated in Table 3, where the slopes of the constructed LFs are given. In Fig. 13 we show the MS LFs of LH 91-I and LH 95. In the left panel we present the LFs of all the MS stars counted within the system's limits of $r \leq 1$ '2 (dashed lines), as well as of the surrounding fields (solid lines) with $1.2<r \leq 2$ '.5 normalized in the same area. These later field LFs were used for the construction of the field subtracted LFs of the systems shown in the right panel of Fig. 13. All LFs presented are completeness corrected.

In Table 3 we present the slopes $s$ of the LFs for various magnitude ranges for all the fields investigated. The magnitude ranges were selected so that the completeness of the corresponding magnitude intervals is in reasonable limits. We show the corresponding minimum completeness limit in Col. 4. We also selected specific magnitude ranges in order to compare the corresponding LF slopes of the systems with the ones of the various field regions. As is shown in Table 3 the slopes of the systems' LFs in the magnitude range of $16.5 \leq B \leq 19.5 \mathrm{mag}$ are systematically shallower than the ones of the fields. The slopes found for LH $91 \&$ LH 95 in the range $13.5 \leq B \leq 19.5 \mathrm{mag}(s=0.18 \&$ $s=0.19$ correspondingly) and for LH 91-I in the range $15.5 \leq B \leq 19.5$ (no brighter star was found within the limits of the system), which was found as $s=0.32$, are very close to the slopes found by Hill et al. (1994) for stellar associations in the LMC for almost the same magnitude ranges. Still, it is worth noting that LH 91-I shows a steeper LF than the ones of the other two systems.

\subsection{Mass functions of the systems and of background field}

A convenient way to characterize the IMF is by the logarithmic derivative $\Gamma$ (Scalo 1986):

$\Gamma=\frac{\mathrm{d} \log \xi(\log m)}{\mathrm{d} \log m}$

$\xi(\log m)$ is the Initial Mass Function, which by definition (Tinsley 1980) is the average number of stars born per year per unit logarithmic (base ten) mass interval per area.

For measuring this quantity for a single stellar system one can assume that all stars in the system are the product of a single star formation event, which is more or less true, especially for young systems like the OB associations. Thus, assuming that all stars in an association were born within the last $10 \mathrm{Myr}$ (which should be the case for our systems) one can refer to the systems' MFs as their IMFs. For the construction of the MFs we performed counts of stars in mass intervals (logarithmic base ten) based on the Geneva evolutionary tracks. We corrected the counted numbers for incompleteness and we normalized them to a surface of $1 \mathrm{kpc}^{2}$. We then applied a linear fit to the relation $\log \xi(\log m)$ (vs.) $\log m$ using a minimum chi-square method. The derived slope is the MF index $\Gamma$. In this nomenclature a Salpeter (1955) IMF corresponds to index $\Gamma=-1.35$. In all cases we limited ourself to stars with $B-V \leq 0.5 \mathrm{mag}$, as the best representative MS population.

The uncertainties in the resulting IMFs of stellar associations are due to the evolutionary tracks between which stars were counted, but the largest uncertainty lies in the 

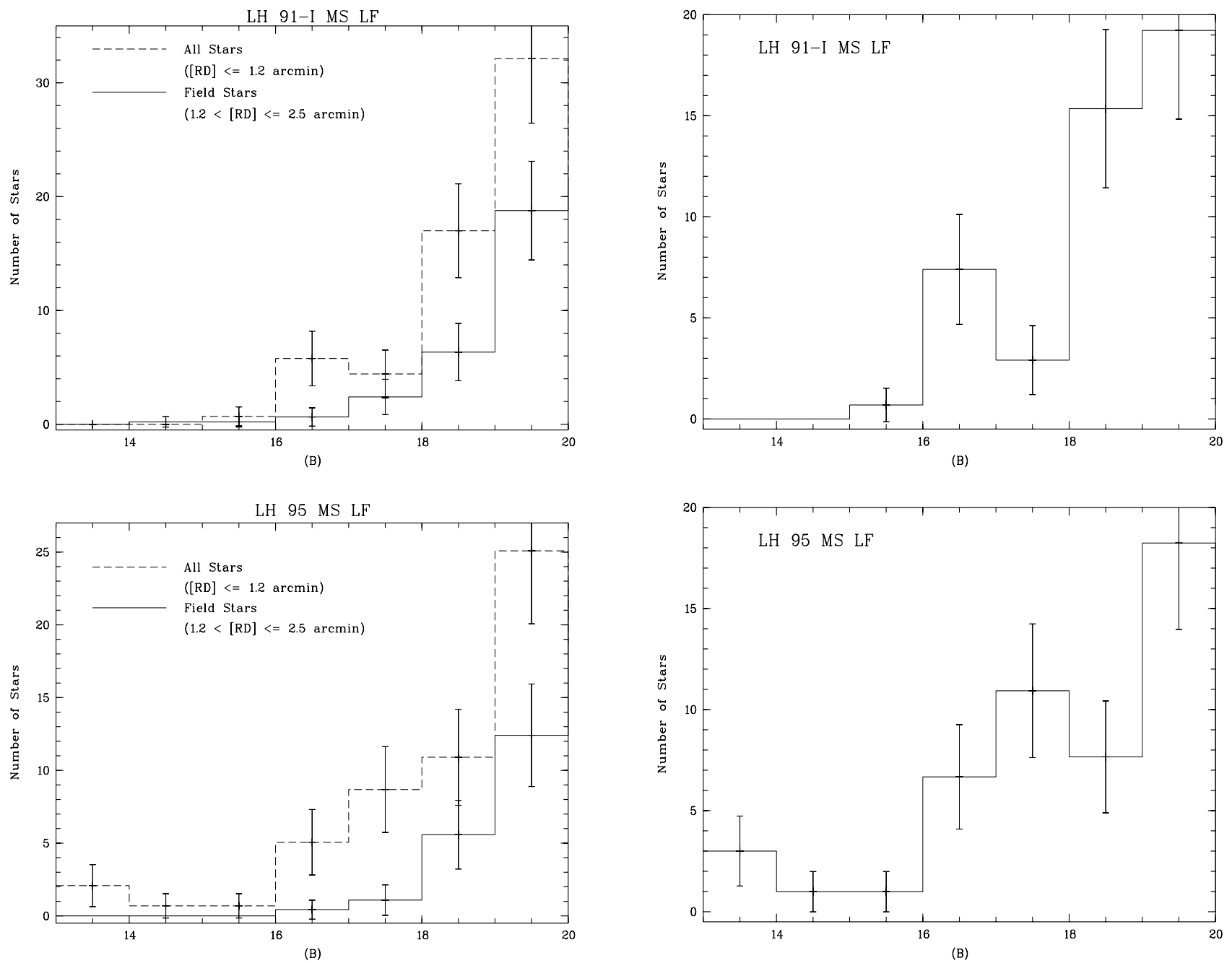

Fig. 13. Left panel: differential LFs for MS stars in the area of LH 91-I and LH 95 (within 1.2) and in the surrounding fields (with radial distances between 1'.2 and 2.5 from each system's centre). All these LFs are corrected for incompleteness and normalized in the same surface of a circle of radius 1.'0. Right panel: differential LFs for MS stars of LH 91-I and LH 95 after field subtraction. The numbers of stars correspond to the area of the systems (surface of a circle of radius 1 '.2).

small stellar sample for such systems, which leads to relatively large Poisson errors especially for the higher-mass bins. In addition, the IMF for massive stars cannot be reliably estimated from photometry alone. Still, the mass range of $1-15 M_{\odot}$ shows to be the best range for the estimation of IMF in star clusters, since the main sequence is well defined, and problems due to pre- or post-main sequence stars are minimized (Scalo 1998).

There are cases, where PMS stars can be present in the LF of clusters even older than $\sim 10 \mathrm{Myr}$ (Belikov \& Piskunov 1997), so the counting of masses for these stars should be based on PMS evolutionary tracks. In the investigation presented here we are limited by the incompleteness to masses above $\sim 2 M_{\odot}$. According to PMS models (Palla \& Stahler 1999) one should expect PMS stars with masses well above this limit, if such a population should exist in the systems. As a consequence, our investigation is restricted to the "evolved" masses in the areas.

In Fig. 14 we present the constructed MFs of LH 91-I and LH 95 within their defined boundaries $(\leq 1$ '2). In the left panel these MFs corrected for incompleteness and normalized to an area of $1 \mathrm{kpc}^{2}$, but not field subtracted, are presented. In the middle panel are shown the constructed MFs of the surrounding fields, as they were defined for the construction of the LFs $(1 ! 2<r \leq 2 ! 5)$. These MFs of the surrounding fields were used for the corresponding field subtracted MFs of the systems, which are shown in the right panel of Fig. 14. The MS MFs of LH 91 and the four empty fields, as well as of background field are shown in Fig. 15.

The slopes of the MFs for various mass ranges are given in Table 4. In order to eliminate the uncertainties mentioned earlier for the construction of the MF, we selected a mass range that corresponds to reasonable completeness in our photometry and which avoids the high mass end. The mass range more suitable given this constraint is about 2 to $11 M_{\odot}$. Still, for most of the empty fields no stars were found with masses higher than about $6 M_{\odot}$. So, for comparison with the background field MFs we also present the MF slopes of the systems and their 
Table 3. LF slopes for the systems, their surounding fields, the 4 empty fields and the general background field, for various magnitude ranges.

\begin{tabular}{|c|c|c|c|}
\hline & $\begin{array}{l}\text { Magnitude } \\
\text { range }\end{array}$ & $\begin{array}{c}\text { LF slope } \\
(s)\end{array}$ & $\begin{array}{c}\text { Completeness } \\
\text { Limit } \\
\end{array}$ \\
\hline \multirow[t]{2}{*}{$\overline{\mathrm{LH} 95^{\star}}$} & $1313.5 \leq B \leq 19.5$ & $0.19 \pm 0.06$ & $35 \%$ \\
\hline & $16.5 \leq B \leq 19.5$ & $0.12 \pm 0.07$ & \\
\hline 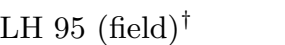 & $16.5 \leq B \leq 19.5$ & $0.51 \pm 0.05$ & $62 \%$ \\
\hline \multirow[t]{2}{*}{ LH 91-I" } & $15.5 \leq B \leq 19.5$ & $0.32 \pm 0.11$ & $35 \%$ \\
\hline & $16.5 \leq B \leq 19.5$ & $0.20 \pm 0.15$ & \\
\hline LH 91-I (field) $)^{\ddagger}$, & $16.5 \leq B \leq 19.5$ & $0.48 \pm 0.02$ & $62 \%$ \\
\hline \multirow[t]{2}{*}{ LH 91} & $13.5 \leq B \leq 19.5$ & $0.18 \pm 0.08$ & $64 \%$ \\
\hline & $16.5 \leq B \leq 19.5$ & $0.47 \pm 0.04$ & \\
\hline & $16.5 \leq B \leq 19.5$ & $0.63 \pm 0.08$ & $62 \%$ \\
\hline Field $2^{\$}$ & $16.5 \leq B \leq 19.5$ & $0.42 \pm 0.14$ & $62 \%$ \\
\hline Field $3^{\ddagger} \S$ & $16.5 \leq B \leq 19.5$ & $0.45 \pm 0.06$ & $62 \%$ \\
\hline Field $4^{\S}$ ' & $16.5 \leq B \leq 19.5$ & $0.61 \pm 0.21$ & $62 \%$ \\
\hline \multirow[t]{2}{*}{ Background Field $^{\ddagger}, \S$} & $13.5 \leq B \leq 19.5$ & $0.41 \pm 0.04$ & $62 \%$ \\
\hline & $16.5 \leq B \leq 19.5$ & $0.54 \pm 0.08$ & \\
\hline \\
\hline \multicolumn{4}{|c|}{$\begin{array}{l}\text { NOTES: The slope does not change significantly for magnitude ranges } \\
\star \star 13.5 \leq B \leq 18.5 \text { and } 13.5 \leq B \leq 17.5\end{array}$} \\
\hline \multirow{2}{*}{\multicolumn{2}{|c|}{$16.5 \leq B \leq 20.5$ and $16.5 \leq B \leq 18.5$}} & & \\
\hline & & & \\
\hline \\
\hline & & & \\
\hline \multicolumn{2}{|c|}{ The slope changes slitely for magnitude } & & \\
\hline \multicolumn{2}{|c|}{ ब $10.0<D<B<18.5$} & & \\
\hline
\end{tabular}

Table 4. MF slopes for the areas of the systems (with and without field subtraction) and their surounding fields, as well as the empty fields and the general Background Field for various mass ranges. All MFs are corrected for incompleteness and normalized to the same surface of $1 \mathrm{kpc}^{2}$. For the cases of LH 91-I and LH 95 we use the note "system + field" for the MF of the areas within 1!2 without field subtraction, the note "field" for the surrounding field of each system $(1.2<r \leq 2$ '.5) and the note "system" for the field subtracted MF of the system $(r \leq 1.2)$. The symbol "-" was used for the cases, where there are several mass bins with zero stars so that the fitted slopes become rather arbitrary.

\begin{tabular}{lcccc}
\hline & \multicolumn{4}{c}{ Mass function slope $(\Gamma)$} \\
& $\sim 2-6 M_{\odot}$ & $\sim 2-8 M_{\odot}$ & $\sim 2-11 M_{\odot}$ & $\sim 2-17 M_{\odot}$ \\
\hline \hline LH 91 & $-2.9 \pm 0.4$ & $-2.2 \pm 0.4$ & $-2.1 \pm 0.3$ & $-2.2 \pm 0.2$ \\
LH 91-I (system + field) & $-2.7 \pm 0.9$ & $-3.3 \pm 0.6$ & $-2.5 \pm 0.6$ & - \\
LH 91-I (field) & $-2.9 \pm 0.4$ & $-2.6 \pm 0.3$ & $-2.5 \pm 0.2$ & $-2.3 \pm 0.2$ \\
LH 91-I (system) & $-2.6 \pm 1.2$ & - & - & - \\
LH 95 (system + field) & $-1.8 \pm 0.2$ & $-1.5 \pm 0.2$ & $-1.8 \pm 0.2$ & $-1.5 \pm 0.2$ \\
LH 95 (field) & $-2.9 \pm 0.4$ & $-3.0 \pm 0.2$ & $-2.9 \pm 0.2$ & - \\
LH 95 (system) & $-1.9 \pm 0.2$ & $-1.3 \pm 0.4$ & $-1.6 \pm 0.3$ & $-1.2 \pm 0.3$ \\
\hline Field 1 & $-3.6 \pm 0.9$ & - & - & - \\
Field 2 & $-4.1 \pm 1.0$ & - & - & - \\
Field 3 & $-4.0 \pm 0.2$ & $-3.7 \pm 0.2$ & - & - \\
Field 4 & $-4.9 \pm 0.6$ & - & - & - \\
Background Field & $-4.2 \pm 0.6$ & $-4.2 \pm 0.4$ & - & - \\
\hline \hline
\end{tabular}

surrounding fields down to this limit. In the MF of LH 91 there is a larger number of stars in the three most massive bins compared to the various fields (Fig. 15). For the mass range of $\sim 2$ to $6 M_{\odot}$ the slope is pretty steep and similar to the MF slopes of the fields surrounding LH 91-I and LH 95. Still, in the area of LH 91 stars with masses up to about $27 M_{\odot}$ were found. Consequently, if we take into account more massive bins the MF is becoming shallower (Table 4).
For the case of LH 91-I, after the field subtraction, the system's MF contains stars up to $\sim 13 M_{\odot}$ and the corresponding slope (with lower mass limit of $\sim 2 M_{\odot}$ ) is the same with the one for the range of $2-11 M_{\odot}$. No stars were found around $\sim 8 M_{\odot}$ in LH 91-I, while stars up to about $17 M_{\odot}$ were found in the surrounding field. For the mass range of $2-6 M_{\odot}$ the MF of LH 91-I, with or without field subtraction, is pretty comparable to the MF of its surrounding field (Fig. 14). LH 95 was found to 


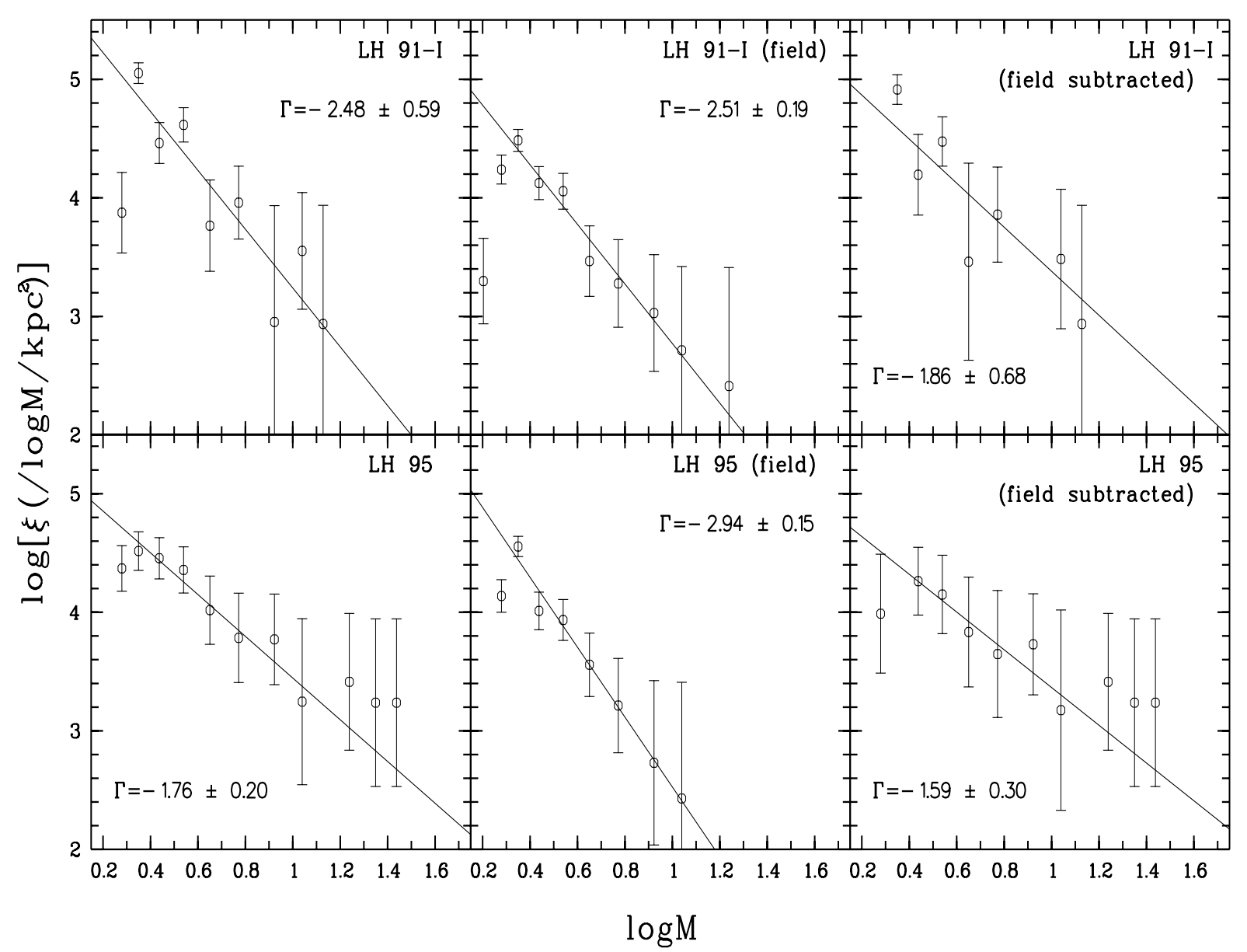

Fig. 14. MFs for MS stars of LH 91-I and LH 95 within their defined areas of radius 1'2 as shown in Fig. 5 (left panels), their surrounding fields (stars with radial distances between 1'.2 and 2.'5 from the systems' centers)(middle panels) and of the systems after subtracting the fields MFs (right panels). The MF of LH 91-I and its surrounding field are rather similar, while after the subtraction of the field MF the slope is shallower but still steeper than -1.35 . On the other hand, the difference of the MF of LH 95 and the one of its surrounding field is obvious. As a consequence the field subtracted MF of the system is much shallower and comparable to the Salpeter slope. All MFs are completeness corrected and normalized to the same surface of $1 \mathrm{kpc}^{2}$. In the case of LH 91-I the slopes are estimated over the mass range of $\sim 2-6 M_{\odot}$, while for LH 95 over the mass range of $\sim 2-11 M_{\odot}$. The linear fits are not weighted.

possess stars in the $\sim 17 M_{\odot}$, as well as in the $\sim 22 M_{\odot}$ and $\sim 27 M_{\odot}$ intervals (before and after the field subtraction). As was expected the MF slope becomes shallower if we take into account these intervals.

In general if we consider all the cases presented here (for masses up to about 8 or 11 and $17 M_{\odot}$ ) we find that the values of the MF slope for LH 91 is centered around $\Gamma=-2.0$ and the one for LH 95 is varying from $\Gamma=-1.6$ to $\Gamma=-1.2$. These limits (considering the various errors) are very close to the IMF slopes found by Massey et al. (1995) for stellar associations in the $\mathrm{LMC}(\Gamma \simeq-1.1 \pm 0.1$ to $-1.7 \pm 0.2)$. They are within the limits of the known IMF slopes for LH associations found by various authors (see e.g. Westerlund 1997 and references therein). On the other hand the MF slopes we found for the field population, which are varying from $\Gamma=-3.7$ up to -4.9 for the mass range of $\sim 2-8 M_{\odot}$ are rather similar to the MF slopes for the massive LMC field stars down to $25 \mathrm{M}_{\odot}$
$(\Gamma=-4.1 \pm 0.2)$ found by Massey et al. (1995) using photometry and spectroscopy. This result is rather interesting, since it implies that the LMC field MF slope does not change significantly towards the less massive end.

\subsubsection{LH 95: Mass segregated or evaporating system?}

The values in Table 4 and Fig. 14 show that the surrounding field of LH 95 has steeper MF slope than that of the system and that the MF slope of the stars in the field surrounding LH 95 is shallower than the slope of the MF of the general background field. Based on this result and on the radial CMDs presented in Sect. 4.1, one may suggest that the difference in the MF slopes of the system and the surrounding field is due to mass segregation (e.g. Kontizas et al. 1998; Fischer et al. 1998; Kontizas et al. 2001). This would mean that the system is extended, but 


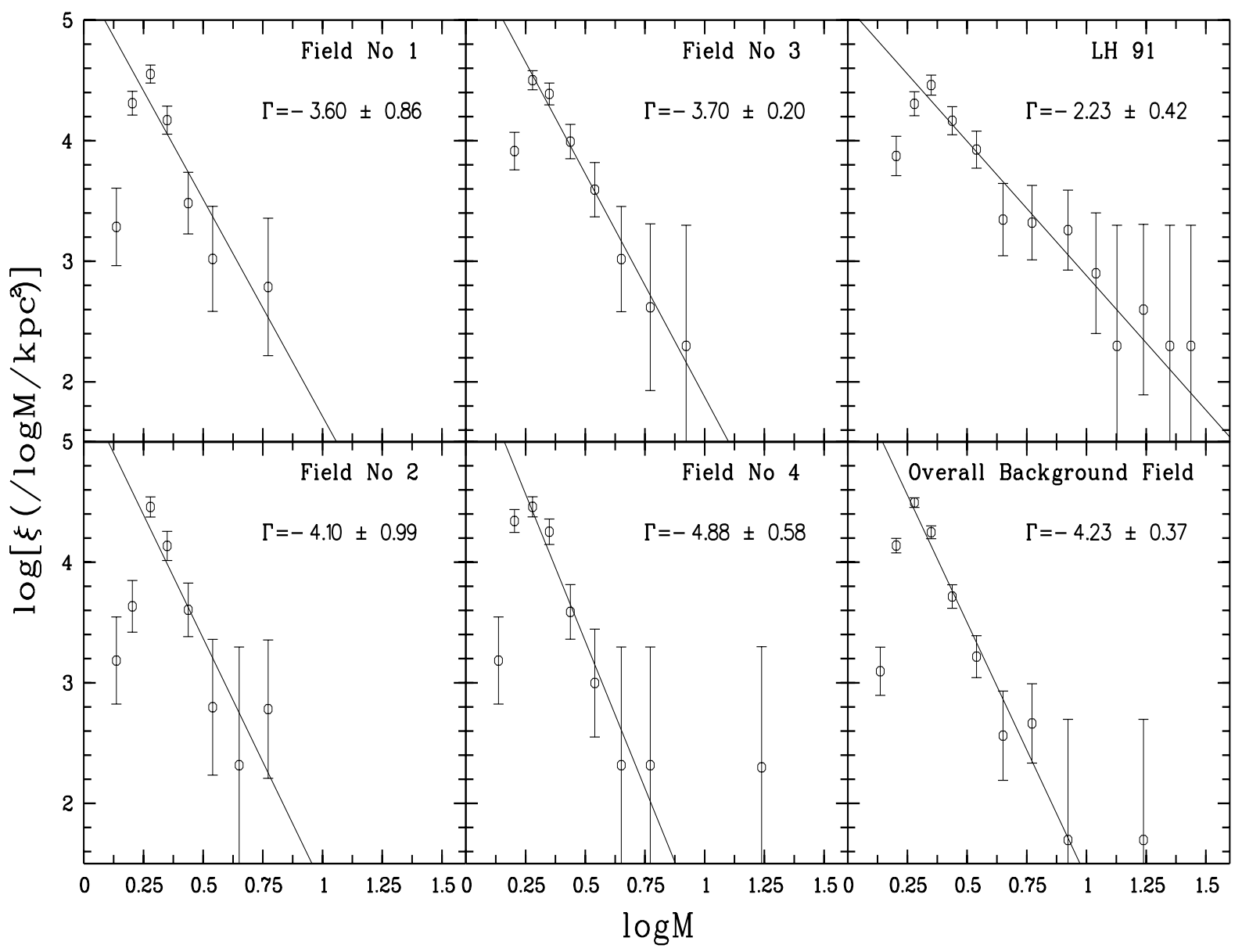

Fig. 15. MFs for MS stars of the 4 empty fields (within the defined area of radius 2'.5 for each field, as shown in Fig. 5). All MFs are completeness corrected and normalized to the same surface of $1 \mathrm{kpc}^{2}$. The MF of the overall background field is shown in the lower-right panel. The slopes presented here are estimated for a mass range of $\sim 2-6 M_{\odot}$ or $\sim 2-8 M_{\odot}$ (see Table 4 ). The linear fits are not weighted.

with its most massive stars located in its centre, as in several open clusters in our Galaxy (Pandey et al. 1992).

There is no direct way to verify the suggestion that LH 95 is an extended mass segregated system. Still there are two facts against it: (1) As is shown in Table 2 the dominant low mass population (17 mag $\leq V \leq 21 \mathrm{mag}$ and $(B-V)<0.6 \mathrm{mag})$ within the system's limits belongs to the system itself. So, the steeper MF of the surrounding field (Fig. 14 middle panel) is not a statistical effect resulting from the higher number of low mass stars within this field than in the system. (2) The system's limits, as were defined in Sect. 3, were based on counts of stars of every magnitude, so that we found the limit where all masses are statistically mixed with a surrounding field population. This means that we were not biased concerning the selection of stars counted according to their mass in order to specify the limits of the system.

Furthermore, taking into account the completeness test, one can see that the completeness for the field population toward low mass is higher than the completeness within the system's area (Fig. 11). This is to be expected, since inside the system crowding is larger and one looses in particular the faint stars. Still, as is shown in Fig. 14, there are more low mass stars within the system's limits than in the surrounding field.

There is also a stronger argument against mass segregation based on the relaxation time of the system. The definition of the half-mass relaxation time, as is generally accepted by several investigators (see e.g. Meylan \& Heggie 1997) is the one presented by Spitzer (1987, Eq. (2$63)$. According to this definition the relaxation time $t_{\mathrm{rh}}$ depends on the total and mean stellar mass, the total number of stars and the half-mass radius. Taking into account the results of the completeness test for LH 95 we estimated these parameters for the area within 1'.2 around the centre of the system (with and without the contribution of field stars) and for the area within 2.5 (see Sect. 6 and Table 5). We found that the relaxation time of LH 95 cannot be less than $35 \mathrm{Myr}$, much larger than the estimated age of the system. This means that the system is not relaxed yet, so dynamical mass segregation can be excluded for LH 95. This suggests that there is a distinction 
between the population of LH 95 and the surrounding field and that the differences in the MF slopes are significant.

We verified the estimated limits of LH 95 by constructing its surface density profile, through star counts in annuli around the system. We estimated, thus, the radius where the density profile becomes flat, as an indication of field population. We performed this estimation using all stars in the area, as well as for selected parts of the system, in order not to take into account the stellar content of the neighboring systems (LH 91 and LH 91-I). The north and west parts seem to be the most "clean" of such populations (see Fig. 5). Using the north and west semicircle, as well as the NW quadrant we found that the limits of LH 95 do not reach distances further than 1'!-1'6. This result is very close to the system's radius of 1.2 , as was found from the star counts. Still, there is a difference between the two results, due to the fact that now we used the north and west parts of the system (to avoid any contamination of stars from LH 91 and LH 91-I), and as is shown in Fig. 3, LH 95 is rather elongated in this direction. So, a radius of $1 ! 4-1 ! 6$ is an overestimation, while the radius of $1 ! 2$ was found by actually measuring the limits, where the density drops below $3 \sigma$ (see Sect. 3.1).

On the other hand one could expect a "mixing" area in the transition zone between the system and its surrounding field, if the system is evaporating. The radius of $\simeq 1.5$ could serve as a limit of this area. These results indicate that LH 95 is probably not a large mass segregated system, but a small young possibly evaporating system. This conclusion is partly supported by the dynamical parameters of the system, as we estimate them in the next section, which give evidence of a loose system, rather experiencing its disruption.

\section{Dynamical characteristics of the systems}

In order to investigate the dynamical behavior of the systems and check for any differences between them we estimated some of their basic dynamical parameters under various assumptions. We estimated the Spitzer radius of each system as the square mean distance of the stars from its centre:

$r_{\mathrm{sp}}=\sqrt{\frac{\sum_{i}^{N} r_{i}^{2}}{N}}$

where $r_{i}$ is the radial distance of the $i$ th star and $N$ the total number of observed stars. Then we found the distance where half of the observed mass is lying (half-mass radius) $r_{\mathrm{h}}$. In both estimations we counted stars within specified limits of the systems. In the case of LH 91 we used all stars in a radius of 2.5 around its centre. For LH 91-I and LH 95 we performed these estimations for the systems' limits as found in Sect. 3 (up to radial distance of 1'.2) and for a larger area around their centers covering their surrounding fields (up to radial distance of 2.5 ). We did this second estimation in order to check what would be the structural properties of these systems if indeed they are covering areas larger than the ones found from the star counts.

In order to estimate the stellar density of the systems (in $M_{\odot} \mathrm{pc}^{-3}$ ) we calculated their masses for three cases: first by converting the observed brightness of each star into mass using the Geneva isochrone grids for 6 to $10 \mathrm{Myr}$, and then by extrapolating the constructed MFs (in Sect. 5.2) down to $\sim 1 M_{\odot}$ and $\sim 0.1 M_{\odot}$. For the two later cases we considered as higher mass limit the $20 M_{\odot}$, since according to the isochrone models the lifetimes of stars with masses larger than this limit are shorter than the age of the systems. So, one should expect too few stars with masses $M>20 M_{\odot}$ to significantly affect the total mass.

This extrapolation was made in order to have a more clear evaluation of the total mass of each system if we assume that stars with masses down to these limits exist, but they were well below our detection limit. The limit of $1 M_{\odot}$ is a reasonable mass threshold for systems as stellar associations. On the other hand we selected as a second test limit the $0.1 M_{\odot}$, since this limit is the smaller detected so far in star forming regions (Brandl et al. 1999). In the cases of LH 91-I and LH 95 we extrapolated their MFs according to the slopes found with and without the contribution of the surrounding fields MFs.

The central stellar density of a stellar system in its half-mass radius is a rather stable dynamical parameter and a good indicator of its stability (e.g. Lada \& Lada 1991). Thus we calculated the stellar density of each system in the half-mass radius as:

$\varrho=\frac{M / 2}{\frac{4}{3} \pi r_{\mathrm{h}}{ }^{3}}$

where $M$ is the total mass.

Finally, in order to have a rough estimation of the time within which each system (in each case) would be disrupted $\left(t_{\mathrm{d}}\right)$ we used Spitzer's (1958) formula, which is based on the assumption that the main cause of the disruption of the systems is their interaction with passing by interstellar clouds:

$t_{\mathrm{d}} \simeq 1.9 \times 10^{8} \varrho\left(\frac{M_{\odot}}{\mathrm{pc}^{3}}\right)$ years.

This result is the product of the assumption that the disruption time of a system depends only on the tidal force of passing interstellar clouds. More specifically $t_{\mathrm{d}}$ was found to be inversely proportional (i) to the total density of clouds averaged over volume (mean density of the interstellar matter) and (ii) to the optical extinction per cloud (mean density of each cloud), which is independent of the cloud's size, and it is proportional (iii) to the mean velocity of the clouds relative to the system (Spitzer 1958, Eq. (14)). The values used by Spitzer were mean density for the interstellar matter of $1 \mathrm{H}$ atom $/ \mathrm{cm}^{3}$, mean density of a typical cloud (of radius $20 \mathrm{pc}$ ) equal to $20 \mathrm{H}$ atoms $/ \mathrm{cm}^{3}$, and a velocity relative to the system of $10 \mathrm{~km} \mathrm{~s}^{-1}$. 
Table 5. Dynamical parameters of the systems. The Spitzer $\left(r_{\mathrm{sp}}\right)$ and half-mass $\left(r_{\mathrm{h}}\right)$ radii (Cols. 2 and 3$)$ have been calculated from the raw star counts. The calculation of the masses by extrapolating the MFs (Cols. 5 and 6 ) are corrected for completeness.

\begin{tabular}{|c|c|c|c|c|c|c|}
\hline \multirow{3}{*}{ System } & \multirow{3}{*}{$\begin{array}{c}\text { Selected } \\
\text { area } \\
\text { radius }\end{array}$} & \multirow{3}{*}{$\begin{array}{c}r_{\mathrm{sp}} \\
(\mathrm{pc}) \\
\end{array}$} & \multirow{3}{*}{$\begin{array}{r}r_{\mathrm{h}} \\
(\mathrm{pc}) \\
\end{array}$} & \multicolumn{3}{|c|}{ Total Mass $\left(\times 10^{3} M_{\odot}\right)$} \\
\hline & & & & \multirow[t]{2}{*}{ Observed $^{\dagger}$} & \multicolumn{2}{|c|}{ down to } \\
\hline & & & & & $\sim 1 M_{\odot}$ & $\sim 0.1 M_{\odot}$ \\
\hline LH 91 & $2 ! 5$ & 26.3 & 22.3 & 1.01 & 2.08 & 23.83 \\
\hline LH 91-I & 1.2 & 12.1 & 9.9 & 0.38 & 1.92 & 45.38 \\
\hline LH 91-I* & & & & & 0.93 & 8.67 \\
\hline LH 91-I & 2.5 & 26.3 & 23.1 & 1.02 & 8.35 & 196.96 \\
\hline LH 95 & $1 ! 2$ & 12.0 & 11.3 & 0.46 & 0.70 & 2.26 \\
\hline LH $95^{\star}$ & & & & & 0.42 & 0.90 \\
\hline \multirow[t]{4}{*}{ LH 95} & 2.5 & 25.5 & 19.3 & 0.94 & 3.03 & 9.79 \\
\hline & \multicolumn{3}{|c|}{$\varrho\left(M_{\odot} \mathrm{pc}^{-3}\right)$} & \multicolumn{3}{|c|}{$t_{\mathrm{d}}(\mathrm{Myr})$} \\
\hline & \multirow[t]{2}{*}{ Observed } & \multicolumn{2}{|c|}{ for masses down to } & \multirow[t]{2}{*}{ Observed } & \multicolumn{2}{|c|}{ for masses down to } \\
\hline & & $\sim 1 M_{\odot}$ & $\sim 0.1 M_{\odot}$ & & $\sim 1 M_{\odot}$ & $\sim 0.1 M_{\odot}$ \\
\hline $\begin{array}{ll}\text { LH } 91 \quad(2.5) \\
\end{array}$ & 0.01 & 0.02 & 0.26 & 1.9 & 3.8 & 49.4 \\
\hline LH 91-I (1'.2) & 0.05 & 0.24 & 5.58 & 9.5 & 45.6 & 1060.2 \\
\hline LH $91-\mathrm{I}^{\star}\left(1{ }^{\prime} \cdot 2\right)$ & & 0.11 & 1.07 & & 20.9 & 203.3 \\
\hline LH 91-I $\left(2{ }^{\prime} .5\right)$ & 0.01 & 0.08 & 1.91 & 1.9 & 15.2 & 362.9 \\
\hline LH $95 \quad$ (1.'2) & 0.04 & 0.06 & 0.19 & 7.6 & 11.4 & 36.1 \\
\hline LH $95^{\star} \quad(1.2)$ & & 0.03 & 0.07 & & 5.7 & 13.3 \\
\hline LH $95 \quad\left(2{ }^{\prime} .5\right)$ & 0.02 & 0.05 & 0.16 & 3.8 & 9.5 & 30.4 \\
\hline \multicolumn{7}{|c|}{$\star$ : In these cases the MF used for the estimation of the expected total mass of each system } \\
\hline
\end{tabular}

The estimated parameters are given in Table 5. These values should be considered only as tentative ones, for the comparison between the systems as far as their dynamical behavior concerns. These values show that the dynamical parameters of the systems are very sensitive to $(i)$ the selected limits of the system and (ii) to the lower mass limit of their stars.

LH 95 is seen to be a loose system with stellar density in the half mass radius not exceeding the value of $0.2 M_{\odot} \mathrm{pc}^{-3}$ and with disruption time of the order of few tens Myr. In the case of the use of the field subtracted MF for the calculation of its total mass it can be shown that the disruption time is rather comparable to the upper limit for the age of the system (if stars of masses down to $0.1 M_{\odot}$ belong to the system), or even smaller (if no stars smaller than $1 M_{\odot}$ belong to the system). This implies that LH 95 could be presently experiencing disruption.

LH 91-I is different, as is shown from its parameters in Table 5 , and can be considered as a bound stellar system with stellar density $\varrho \simeq 6 M_{\odot} \mathrm{pc}^{-3}$ if it contains stars as small as $0.1 M_{\odot}$ within 1.2 and a corresponding disruption time of the order of a Gyr. If one considers the MF field subtracted for the calculation of the expected total mass of the system down to $0.1 M_{\odot}$ and that the system is not larger than $1 ! 2$, then still its disruption time is larger than the estimated age. In the case where the system extends further out to say 2.5 with a stellar content of masses not smaller than $1.0 M_{\odot}$, its density becomes much smaller $\left(\lesssim 0.1 M_{\odot} \mathrm{pc}^{-3}\right)$ and its disruption time is comparable to its age. Our observations, so far, favor the case where the system is concentrated into 1.2 , but still there are no indications about the lower mass end of its stellar population, in order to have a clear estimation of the total mass of the system and subsequently of its disruption time. In addition the fact that the system is very young containing few intermediate mass Be stars implies that pre-main sequence stars with masses down to $0.1 M_{\odot}$ could belong to the system. Such stars have been identified in the Galaxy clustered around Herbig Ae/Be stars (e.g. Testi et al. (1998). It should be noted that this might also be the case of LH 95, where we identify Be stars as well.

Finally LH 91 is seen from its estimated parameters to be an unbound system with stellar density not larger than $\sim 0.3 M_{\odot} \mathrm{pc}^{-3}$ and a disruption time varying from less than 5 Myr up to few tens of Myr. The existence of diffuse only Hu emissivity and the loose appearance of the system favors the case where the disruption time is rather small $\left(t_{\mathrm{d}} \simeq 4 \mathrm{Myr}\right)$.

\section{Discussion}

In this section we first summarize published characteristics of LH 91 and LH 95. Our purpose is to use this information along with our results in order to check the differences between these systems in terms of their physical properties.

Smith et al. $(1987,1990)$ observed the LMC in two ultraviolet bandpasses centered near $1500 \AA\left(m_{1500}\right)$ and $1900 \AA\left(m_{1900}\right)$, and they concentrated their analysis on the associations by Lucke \& Hodge (1970). They verified 

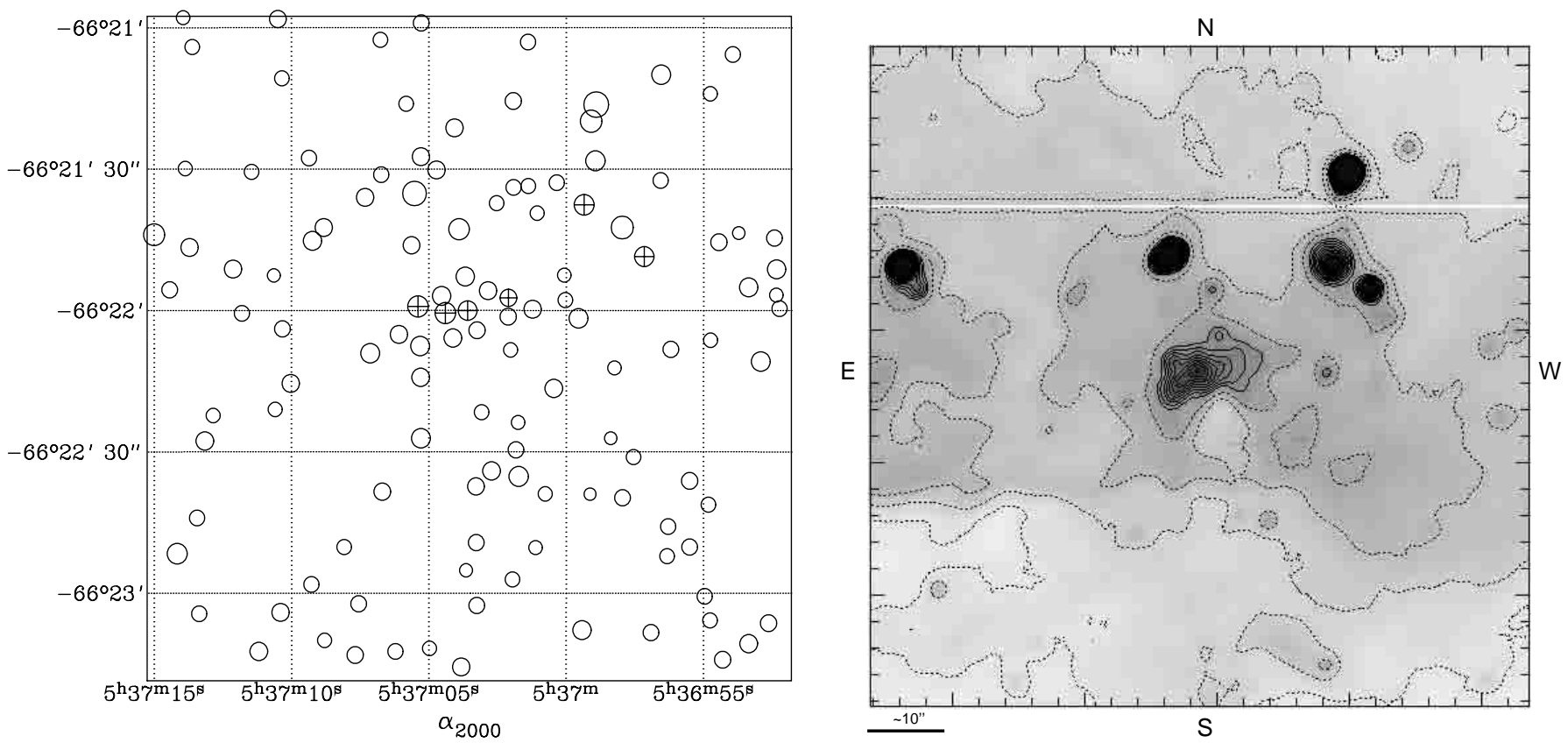

Fig. 16. Star chart of the sources found in optical (left) and H $\alpha$ (right) passbands in the area around LH 95. The crossed circle symbols $(\oplus)$ represent the locations of the Be stars.

that the fainter an association is in the UV, the lower the proportion of bluest stars among its members should be. They came to this conclusion by plotting $m_{0(1500)}$ versus $m_{0(1500)}-m_{0(1900)}$ in terms of magnitude and corrected for reddening (their Fig. 3b).

These authors found magnitude $m_{0(1900)}=8.5$ and colour $m_{0(1500)}-m_{0(1900)}=-0.4$ for LH 95. These values place the system in the left of the expected main sequence slope for spectral types B0-A5 in their plot $m_{0(1500)}$ (vs.) $m_{0(1500)}-m_{0(1900)}$. This suggests that LH 95 is among the richer in early type stellar content associations. The corresponding values for LH 91 are $m_{0(1900)}=8.6$ and $m_{0(1500)}-m_{0(1900)}=0.6$, which classify LH 91 among the poorer in early type stellar content associations.

They also estimated the ionizing flux emitted by the associations and they provide their Lyman line continuum fluxes. The flux of LH 95 as was estimated by Smith et al. (1990) is $F_{\mathrm{Ly}}=24.8 \times 10^{48}$ photons $\mathrm{s}^{-1}$, while the one of LH 91 was found to be $9.0 \times 10^{48}$ photons $\mathrm{s}^{-1}$. The FUV fluxes as estimated by Page \& Carruthers (1981a, $1981 b)$ is $F_{\mathrm{FUV}} \simeq 139 \times 10^{-8} \mathrm{erg} \mathrm{s}^{-1} \mathrm{~cm}^{-2}$ for LH 95 and $F_{\mathrm{FUV}} \simeq 1.7 \times 10^{-8} \mathrm{erg} \mathrm{s}^{-1} \mathrm{~cm}^{-2}$ for LH 91 .

These numbers suggest that while LH 95 is a system rich in early type stars with high Lyman continuum and UV fluxes, LH 91 is a much less "energetic" system. More quantitavely speaking, it is shown that LH 95 emits about 3 times more Lyman flux than LH 91, while the FUV emission seems to be about 82 times higher for LH 95 than for LH 91. Braunsfurth \& Feitzinger (1983) have compiled data for each of the LH stellar associations and their related emission regions. They show that the emission of the HII region DEM L 252 around LH 95 is about 6 times higher than the one around LH 91 (DEM L 251). We compared the $\mathrm{H} \alpha$ intensities of our observations for the areas of these HiI regions and we found that the total $\mathrm{H} \alpha$ intensity of DEM L 252 is about 3 times higher than the corresponding intensity of DEM L 251, while the mean intensity (per pixel) shows to be about 2 times higher than the one of DEM L 251. Braunsfurth \& Feitzinger (1983) assumed mean diameters of the emission regions of $71 \mathrm{pc}$ for DEM L 251 and 80 pc for DEM L 252, which are in very good agreement with our estimations of the sizes of these regions from the $2 \sigma \mathrm{H} \alpha$ map of the area (Fig. $2-$ right panel).

Interesting results concerning these two systems came from Wang \& Helfand (1991), who carried out a study of the X-ray properties of $86 \mathrm{LH} \mathrm{OB}$ stellar associations, using data from the Einstein Observatory (Giacconi et al. 1979). They examined the coincidence of discrete X-ray sources previously tabulated by Wang et al. (1991), with these associations and they found $22 \mathrm{X}$-ray sources within 17 associations. They also identified six additional LH associations with diffuse X-ray emission. LH 91 \& LH 95 do not belong in either of these two groups. Wang \& Helfand (1991) define these systems as undetected, meaning that there are no indications of discrete X-ray sources, such as $\mathrm{X}$-ray binaries or bright young SNRs above the Einstein detection limit.

In addition, Chu et al. (1994), in order to investigate the possibility of using the interstellar absorption properties in the UV as diagnostic of hidden SNR shocks, used the IUE archive to study selected objects, most of them members of Lucke \& Hodge stellar associations. Among the 33 most suitable for study is Sk -66 172 
(Sanduleak 1969), an O5 V star (Conti et al. 1986) in the area of LH 95. They measured the centroid velocities of the interstellar high-ionization (averaged Civ, Sirv), as well as the low-ionization (averaged SII, SiII \& CII ${ }^{\star}$ ) lines, in order to study their difference, as a clear indication of the presence of SNR shocks in X-ray superbubbles. This difference for Sk -66172 is of the order of $\simeq 20$, not addequate to interpret it as a diagnostic for a SNR shock. It shows, in spite of large amounts of CIV and Sirv detected, that these lines likely are produced in the HiI region directly irradiated by a hard radiation field rather, than in a pervasive hot gaseous halo around the LMC. So, there is clear evidence that there was no SN explosion in the areas of LH 91 and LH 95, at least for the last few million years. This fits to the absence of SNR structures in our $\mathrm{H} \alpha$ maps.

\subsection{The case of $L H 95$ (DEM L 252)}

We found that LH 95 is a young stellar association lying in the most energetic portion of the surrounding HiI region DEM L 252. By the time of their formation OB stars (with masses larger than $3 M_{\odot}$ ) change radically the physical conditions of their environment due to their strong stellar winds and their large amount of UV radiation, which ionizes the surrounding interstellar gas (Kunze 1991). The result will be an HiI region, which will expand into the ISM until the source of ionizing radiation vanishes due to stellar evolution. Another energy release mechanism, which impact the surrounding environment of OB stars, is SN explosions.

In Fig. 16 we show the star chart of the area $2 ! 5 \times 2 ! 5$ around the centre of LH 95 . We also present the corresponding grayscale $\mathrm{H} \alpha$ map with the most energetic regions ( $3 \sigma$ above the local field) shown with thick contour lines. From this figure is shown that the radiation of DEM L 252 is highly connected to the emission of the very central area of LH 95 .

Massive stars are born in dense cores of molecular clouds (clumps). As these stars evolve, after the complete ionization of their clumps, the HII region expands further out into the molecular cloud on scales $\lesssim 50$ pc. During this procedure many clumps embedded in the cloud are overtaken and crush while they photoevaporate (Bertoldi 1989). According to Yorke (1986), when the expanding HII region arrives at the edge of molecular cloud a champagne flow develops due to the pressure gradient between cloud and ambient medium. This leads to the destruction of the molecular cloud. The $\mathrm{H} \alpha$ image of Fig. 2 seems to show such a champagne flow around LH 95. If this is the case then what we actually see in the $\mathrm{H} \alpha$ map is the initial molecular cloud, in the core of which LH 95 was born.

The ionization front of the HiI regions runs towards the Strömgren radius with extremely large (almost sonic) velocities. The Strömgren radius characterizes the volume of nearly complete ionization of a uniform-density surrounding medium. The ionization front diameter of an evolved HiI region is (Spitzer 1980):

$D_{\mathrm{H} \mathrm{II}}(t)=2 R_{\mathrm{s}}\left(1+\frac{c_{\mathrm{s}} \cdot t}{R_{\mathrm{s}}}\right)^{0.571}$

where $c_{\mathrm{S}}$ is the velocity of sound in the ionized medium $\left(\simeq 10-13 \mathrm{~km} \mathrm{~s}^{-1}\right)$. The initial Strömgren radius $R_{\mathrm{s}}$ is:

$R_{\mathrm{s}}=\left(\frac{3}{4 \pi} \cdot \frac{S_{\star}}{n_{0}^{2} \beta}\right)^{\frac{1}{3}}$

where $S_{\star}$ is the Lyman continuum flux, $n_{0}$ the uniform density of the ambient medium (mostly $\mathrm{H}$ ) and $\beta$ is the total recombination coefficient above the ground level (Osterbrock 1989) $\beta \simeq 2 \times 10^{-19} \mathrm{~m}^{3} \mathrm{~s}^{-1}$.

A typical B0 V star emits about $10^{48}$ photons $\mathrm{s}^{-1}$ (Schaerer \& de Koter 1997) with a Strömgren radius of $R_{\mathrm{s}} \simeq 1.65 \mathrm{pc}$ (Vacca et al. 1996). The corresponding values for a typical O5 V star are $F_{\mathrm{Ly}} \simeq 10^{49.5}$ photons s $^{-1}$ and $R_{\mathrm{s}} \simeq 4.72$ pc. Using Eq. (7) we find that the corresponding HiI regions will have an ionization front diameter of $\sim 26 \mathrm{pc}$ for the B0 V star and $\sim 42 \mathrm{pc}$ for the $\mathrm{O} 5 \mathrm{~V}$ star in a period of $5 \mathrm{Myr}$. The later diameter is more or less equal to the size of LH 95 as was found from our star counts $(\sim 2 ! 4)$. This means that a single O5 V star is able to ionize the gas around it up to the limits of the system within a short period of about $5 \mathrm{Myr}$.

In addition, if we assume that the time needed for the whole HiI region to expand in a diameter equal to the one we found for DEM L $252(\sim 75 \mathrm{pc})$ is similar to the upper limit of the age of the system $(\sim 10 \mathrm{Myr})$, then solving Eq. (7) for $R_{\mathrm{s}}$, we derive an initial Strömgren radius equal to $\simeq 15$ pc. If we use the Lyman flux found for LH 95 by Smith et al. (1990) in Eq. (8) we find that the density of the ambient medium in the region is $n_{0} \simeq 17 \mathrm{~cm}^{-3}$. Assuming longer expansion time $(\sim 15 \mathrm{Myr})$, the initial Strömgren radius is found to be smaller $\left(R_{\mathrm{s}} \simeq 9.6 \mathrm{pc}\right)$ and the ambient medium almost two times denser $\left(n_{0}=\right.$ $33 \mathrm{~cm}^{-3}$ ). The density values we found for the ambient medium in DEM L 252 are at least three times larger than the values derived for the emitting gas in the HiI region $\mathrm{N} 51 \mathrm{D}$ in the LMC from $\mathrm{H} \alpha$ measurements by Lasker (1980) and from $\mathrm{H} \beta$ by Dickel et al. (1964) $\left(n(e) \sim 6 \mathrm{~cm}^{-3}\right)$.

A final test can be applied if we consider that the limits of the system can represent the initial Strömgren radius of the HiI region $\left(R_{\mathrm{s}} \simeq 19 \mathrm{pc}\right)$. Then the time needed for DEM L 252 to expand up to a diameter of about $75 \mathrm{pc}$ is found from Eq. (7) to be almost $4 \mathrm{Myr}$ and from Eq. (8) we find that the density of the ambient medium is equal to $n_{0} \simeq 11 \mathrm{~cm}^{-3}$. Another interesting feature in the $\mathrm{H} \alpha$ images of LH 95 is the blanket-like feature on the south part of DEM L 252. In Fig. 16 this feature is represented by the southern low radiation (light gray) contours, while it is more apparent in Fig. 4 (top left panel) of the $\mathrm{H} \alpha$ map 5!0 $\times 5 ! 0$ around LH 95. Cloudlets projected in front and just below LH 95, probably members of the parental molecular cloud could produce such a phenomenon. 


\subsection{The case of $L H 91$ (DEM L 251) \& LH 91-I}

Solving Eqs. (7) and (8) for LH 91, assuming that the time needed for the whole HiI region to expand in a diameter equal to $\sim 65 \mathrm{pc}$ (as we found from the $1 \sigma \mathrm{H} \alpha$ image of DEM L 251 - Fig. 4) is also 10 to $15 \mathrm{Myr}$, and using the Lyman continuum found for this system by Smith et al. (1990) we find that the initial Strömgren radius of this HII region should be equal to $R_{\mathrm{s}} \simeq 7$ to $11 \mathrm{pc}$. Consequently, the density of the ambient medium is found to be similar to the values found for DEM L 252 and between 16 and $32 \mathrm{~cm}^{-3}$. This conclusion suggests that, as far as the density of their ambient medium is concerned, both DEM L 251 and DEM L 252 are comparable to each other, the later being brighter in $\mathrm{H} \alpha$.

LH 91-I, finally does not seem to be like either LH 91 or LH 95. In this case the ionization is directly connected to the emission stars (as in LH 95), but does not show any expanding front, or any larger structure in the $\mathrm{H} \alpha$ map of the area (Fig. 4). A rather interesting feature is the bright Be star to the south-east side of this image, which show strong emissivity. If this star is a Herbig emission star (NIR observations needed for such a verification), then it could be a good target for the detection of PMS population clustered around it.

\section{Conclusions}

We report on the results of our optical photometric study of three stellar associations located on the north-east edge of the super-giant shell LMC 4 in the Large Magellanic Cloud. The investigation of such systems may provide additional information on the stellar content at the extremities of super-giant shells and consequently on the formation history of such structures.

We observed an area about $20.5 \times 20.5$ centered on LH 95 (Lucke \& Hodge 1970) in BVR and $\mathrm{H} \alpha$ bands. This area covers also LH 91 (Lucke \& Hodge 1970) and LH 91-I (Kontizas et al. 1994). The $R$ and $\mathrm{H} \alpha$ observations were used in order the emission line stars population to be identified. Be stars were found to belong in all three systems, as well as to the general background field. The most interesting case is LH 95, where we verify that the highest $\mathrm{H} \alpha$ emissivity in DEM L 252 (the related large bright HII region) is connected to four Be stars concentrated in the centre of the system. The $\mathrm{H} \alpha$ emission of LH 91-I is also related to the Be stars identified within the limits of the system. In LH 91 only two Be stars were identified. The corresponding HII region (DEM L 251) seems to be only faintly related to these stars, since they are located both to the south-west side of the region. In addition DEM L 251 is seen to be a rather diffuse or disrupted His region in contrast to DEM L 252.

We constructed the CMDs of all three systems as well as of their surrounding fields. From their radial CMDs it was found that LH 95 contains a bright MS population, while the surrounding field is mostly characterized by redder and fainter stellar populations. This is not the case for
LH 91-I, which actually shows to be embedded in a young field population. Using isochrone fits we specify the age limits of the systems and of background field. All systems are younger than $10 \mathrm{Myr}$. This result supports the results by Braun et al. (1997, 2000), who show that there is no age gradient inside LMC 4, but they would expect younger populations on the edge of the shell. Specifically, our results seem to agree with the age estimations of various authors for systems on the edge of LMC 4 (e.g. Sagar \& Richtler 1991; Petr et al. 1994; Will et al. 1996; Olsen et al. 1997), which show a small range of $\simeq 5$ to 15 Myr.

The background field on the other hand is significantly older at $\geq 50 \mathrm{Myr}$. The reddening toward the systems was found to vary between $E(B-V) \simeq 0.15$ and 0.20 . These values are in good agreement with estimations of the reddening toward other young stellar systems in the LMC and in the general region of LMC 4 (e.g. Laval et al. 1986; Will et al. 1997; Olsen et al. 1997).

We constructed the Luminosity and Mass Functions of the systems and of the various fields. We found that the LF slopes of the systems are systematically shallower than the ones of the surrounding and the more distant field regions, and they are comparable to the LF slopes found earlier for LH associations by other authors (e.g. Hill et al. 1994), varying from $s \simeq 0.12$ to 0.32 . The systems' MF slopes were also found to be more shallow than those of the field regions. These slopes were found to belong to the range of $\Gamma \simeq-1.0$ to -2.0 , comparable to the $\mathrm{MF}$ slopes already found for other stellar associations in the LMC. The MF slope for the background field of the area was found around $\Gamma \simeq-4.0$. These values are very close to the slopes found for massive stars of the LMC field by Massey et al. (1995).

We performed several tests in order to estimate the dynamical parameters of the systems under various assumptions. We conclude that while LH 91 appears to be a loose system, rather disrupted, LH 91-I shows a set of structural parameters varying extremely with the assumptions used for their calculation. So, if we assume that no stars with masses smaller than about $1 M_{\odot}$ belong to LH 91-I, then the system behaves like an unbound system very close to disruption. On the other hand if stellar masses down to $0.1 M_{\odot}$ are members of LH 91-I, then the system seems to be a bound young cluster. The existence of Be stars within the limits of this system could suggest that pre-main-sequence population (down to this mass range) belongs to it. This suggestion is supported by the detection of PMS stars clustered around Herbig Ae/Be stars by Testi et al. (1998).

Finally LH 95 was shown to be a loose system very close to, or undergoing disruption. The comparison of the stellar content and the MF of the system with the surrounding field suggests that the system could be larger in diameter than $\simeq 3$ '. 0 experiencing mass segregation (with its more massive stars centrally concentrated). We found that this does not seem to be the case, since we verified that the radius of the system is not larger than $\simeq 1.5$ and that LH 95 is a rather disrupted system. In addition 
more low mass stars are seen to be located within the system's limits, than in the surrounding field, showing that all masses are concentrated within 1'.2 around LH 95 and not only the larger ones. All these suggest that there is a good distinction between the stellar populations of LH 95 and its surrounding field, as well as between this field and the general background field population (due to their different MF slopes). This consequently may imply that LH 95 is evaporating, in the sense that the system feeds the general background field with less massive stars, which are escaping through its surrounding field, resulting a shallower MF slope for this field than the one of the more distant field regions.

Acknowledgements. Dimitris Gouliermis is grateful for the support from the Deutsche Forschungsgemainschaft (DFG), in the framework of the Graduiertenkolleg "The Magellanic system and other dwarf galaxies" (GRK 118/2-96).

\section{References}

Alcock, C., Allsman, R. A., Axelrod, et al. 1996, AJ, 111, 1146 Alongi, M., Bertelli, G., Bressan, A., et al. 1993, A\&AS, 97, 851

Belikov, A. N., \& Piskunov, A. E. 1997, ARep, 41, 28

Bertoldi, F. 1989, ApJ, 346, 735

Brandl, B., Brandner, W., Eisenhauer, F., et al. 1999, A\&A, $352,69 \mathrm{~L}$

Braun, J. M., Bomans, D. J., Will, J.-M., \& de Boer, K. S. 1997, A\&A, 328, 167

Braun, J. M., de Boer, K. S., \& Altmann, M. 2000, A\&A, Submitted

Braunsfurth, E., \& Feitzinger, J. V. 1983, A\&A, 127, 113

Caputo, F. 1997, MNRAS, 284, 994

Chu, Y.-H. 1998, in The Magellanic Clouds and Other Dwarf Galaxies, Proceedings of the Bonn/BochumGraduiertenkolleg Workshop, ed. T. Richtler, \& J. M. Braun (Aachen: Shaker Verlag), 11

Chu, Y.-H., Wakker, B., Mac Low, M.-M., \& Garcia-Segura, G. 1994, AJ, 108, 1696

Conti, P. S., Garmany, C. D., \& Massey, P. 1986, AJ, 92, 48

Davies, R. D., Elliott, K. H., \& Meaburn, J. 1976, MmRAS, 81,89

de Boer, K. S., Braun, J. M., Vallenari, A., \& Mebold, U. 1998, A\&A, 329, L49

Dickel, H. R., Aller L. H., \& Faulkner, D. J. 1964, in The Galaxy and the Magellanic Clouds, ed. F. J. Kerr, \& A. W. Rodgers (Canberra: Australian Acad. Sci.), IAU Symp., 20, 294

Dolphin, A. E., \& Hunter, D. A. 1998, AJ, 116, 1275

Dopita, M. A., Mathewson, D. S., \& Ford, V. L. 1985, ApJ, 297,599

Efremov, Yu. N., \& Elmegreen, B. G. 1998, MNRAS, 299, 643

Efremov, Yu. N., Elmegreen, B. G., \& Hodge, P. W. 1998, ApJ, 501, 163

Fischer, P., Pryor, C., Murray, S., et al. 1998, AJ, 115, 592

Giacconi, R., Branduardi, G., Briel, U., et al. 1979, ApJ, 230, 540

Grebel, E. K., Richtler, T., \& de Boer, K. S. 1992, A\&A, 254, L5

Grebel, E. K. 1997, A\&A, 317, 448

Henize, K. G. 1956, ApJS, 2, 315
Hodge, P. W. 1986, in Luminous Stars and Associations in Galaxies, Luminous Stars and Associations in Galaxies, ed. C. de Loore, A. Willis, \& P. Laskarides (Dordrecht: Reidel), IAU Symp., 116, 369

Hill, R. J., Madore, B. F., \& Freedman, W. L. 1994, ApJ, 429, 192

Hiltner, W. A., \& Johnson, H. L. 1956, ApJ, 124, 367

Keller, S. C., Wood, P. R., \& Bessell, M. S. 1999, A\&AS, 134, 489

Kim, S., Staveley-Smith, L., Sault, R. J., Kesteven, M. J., McConnell, D., \& Freeman, K. C. 1997, PASA, 14, 119

Kim, S., Dopita, M. A., Staveley-Smith, L., \& Bessell, M. S. 1999, AJ, 118, 2797

Koornneef, J. 1983, A\&A, 128, 84

Kontizas, M., Kontizas, E., Dapergolas, A., Argyropoulos, S., \& Bellas-Velidis, I. 1994, A\&AS, 107, 77

Kontizas, M., Hatzidimitriou, D., Bellas-Velidis, I., et al. 1998, A\&A, 336, 503

Kontizas, M., Keller, S. C., Gouliermis, D., et al. 2001, MNRAS, submitted

Kunze, R. 1991, in AG Rev. Mod. Astron. 6, Stellar Evolution and Interstellar Matter, ed. G. Klare (Hamburg: Astronomische Gesellschaft), 257

Lada, C. J., \& Lada, E. A. 1991, in The Formation and Evolution of Star Clusters, ed. K. Janes (San Francisco: ASP), ASP. Conf. Ser., 13, 3

Landolt, A. U. 1992, AJ, 104, 372

Lasker, B. M. 1980, ApJ, 239, 65

Laval, A., Greve, A., \& van Genderen, A. M. 1986, A\&A, 164, 26

Leitherer, C., \& Wolf, B. 1984, A\&A, 132, 151

Lucke, P. B. 1974, ApJS, 28, 73

Lucke, P. B., \& Hodge, P. W. 1970, AJ, 75, 171

Madore, B. F. 1982, ApJ, 253, 575

Massey, Ph., Lang, C. C., DeGioia-Eastwood, K., \& Garmany, C. R. 1995, ApJ, 438, 188

McGee, R. X., \& Milton, J. A. 1966a, Au. J. Phys., 19, 343

McGee, R. X., \& Milton, J. A. 1966b, Au. J. Phys., Ap. Suppl., No 2

Meaburn, J. 1981, in ASSL vol. 91, Investigating the Universe, ed. F. Kahn (Dordrecht: Reidel), 61

Mihalas, D., \& Binney, J. 1981, Galactic Astronomy (San Francisco: Freeman)

Oestreicher, M. O., Gochermann, J., \& Schmidt-Kaler, T. 1995, A\&AS, 112, 495

Oey, M. S. 1999, in New Views of the Magellanic Clouds, ed. Y.-H. Chu et al. (San Francisco: ASP), IAU Symp., 190, 78

Olsen, K. A. G., Hodge, P. W., Wilcots, E. M., \& Pastwick, L. 1997, ApJ, 475, 545

Olson, B. I. 1975, PASP, 87, 349

Osterbrock, D. E. 1989, Astrophysics of Gaseous Nebulae and Active Galactic Nuclei (Mill Valley: University Science Books)

Page, Th., \& Carruthers, G. R. 1981a, ApJ, 248, 906

Page, Th., \& Carruthers, G. R. 1981b, ApJ, 260, 413

Palla, F., \& Stahler, S. W. 1999, ApJ, 525, 772

Pandey, A. K., Mahra, H. S., \& Sagar, R. 1992, BASI, 20, 287

Petr, M., Bomans, D. J., \& Grebel, E. K. 1994, in ESO Conf. Proc., 51, 3rd CTIO/ESO Workshop on the Local Group, ed. A. Layden, R. C. Smith, \& J. Storm, 86

Ratnatunga, K. U., \& Bahcall, J. N. 1985, ApJS, 59, 63

Sagar, R., \& Richtler, T. 1991, A\&A, 250, 324 
Salpeter, E. E. 1955, ApJ, 121, 161

Sanduleak, N. 1969, CTIO Contrib. No., 89

Scalo, J. M. 1986, Fund. Cosmic Phys., 11, 1

Scalo, J. M. 1998, in The Stellar Initial Mass Function, ed. G.

Gilmore, \& D. Howell (San Francisco: ASP), ASP. Conf. Ser., 142, 201

Meylan, G., \& Heggie, D. C. 1997, A\&AR, 8, 1

Schaerer, D., Meynet, G., Maeder, A., \& Schaller, G. 1993, A\&AS, 98, 523

Schaerer, D. \& de Koter, A. 1997, A\&A, 322, 598

Smith, A. M., Cornett, R. H., \& Hill, R. S. 1987, ApJ, 320, 609

Smith, A. M., Cornett, R. H., \& Hill, R. S. 1990, ApJ, 355, 746

Spitzer, L. Jr. 1958, ApJ, 127, 17

Spitzer, L. Jr. 1980, Physical Processes in the Interstellar Medium (New York: Wiley-Interscience)

Spitzer, L. Jr. 1987, Dynamical Evolution of Globular Clusters (Princeton: Princeton University Press)
Stetson, P. B. 1987, PASP, 99, 191

Testi, L., Palla, F., \& Natta, A. 1998, A\&AS, 133, 81

Tinsley, B. M. 1980, Fund. Cosmic Phys., 5, 287

Turner, D. G. 1976, AJ, 81, 1125

Vacca, W. D., Garmany, C. D., \& Shull, J. M. 1996, ApJ, 460, 914

Wang, Q. \& Helfand, D. J. 1991, ApJ, 373, 497

Wang, Q., Hamilton, T., Helfand, D. J., \& Wu, X. 1991, ApJ, 374,475

Westerlund, B. E. \& Mathewson, D. S. 1966, MNRAS, 131, 371

Westerlund, B. E. 1997, The Magellanic Clouds, Cambridge Astrophysical Series (Cambridge University Press)

Will, J.-M., Bomans, D. J., Vallenari, A., Schmidt, J. H. K., \& de Boer, K. S. 1996, A\&A, 315, 125

Will, J.-M., Bomans, D. J., \& Dieball, A. 1997, A\&AS, 123, 455

Yorke, H. W. 1986, ARA\&A, 24, 49 